\title{
Error Correction of Quantum Channels
}

\author{
by
}

\author{
Mona Meghdari Miardan
}

A thesis submitted to the Faculty of Graduate and Postdoctoral Affairs in partial fulfillment of the requirements for the degree of

Master of Science

in

Pure Mathematics

Carleton University Ottawa, Ontario

(C) 2014

Mona Meghdari Miardan 


\section{Abstract}

As manifested in Shor's groundbreaking seminal work, quantum mechanics promise the possibility of having substantially more effective computation devices. This is in fact the result of quantum parallelism: the coherent interference pattern between the multitude of superpositions.

But the fragility of a quantum state, which on one hand is used to take advantage of the power of entanglement, also can result in undesired interference between the state of the quantum system, carrying or storing the information, with the environment. The problem of maintaining quantum coherence remains one of the most important obstacles in the attempt of exploiting the new possibilities opened up by applications of quantum mechanics in classical computations.

Redundancy, which is the main tool in all classical error correcting schemes is no more available in quantum setting, because of the no-cloning theorem, that prevents the duplication of quantum states.

The seminal independent work of Shor [32] and Steane [35] gave birth to the current active theory of quantum error correction, which is the subject of this thesis.

This thesis is a non-technical introduction to the theory of quantum error correction. We present the mathematical formalism for the theory, covering the main ideas of correcting schemes and techniques, laid in a mathematical framework, avoiding unnecessary computational and physical technicalities. 


\section{Acknowledgements}

Foremost, I would like to thank my supervisors Dr. Mehrdad Kalantar and Dr. Matthias Neufang for their priceless guidance, support and encouragement provided throughout my graduate studies. I am more than thankful to these wonderful professors for their time and effort devoted during these two years.

I would also like to thank Dr. Kennedy for his kind support and help during these years. I also want to thank the School of Mathematics and Statistics and their kind staff.

Finally, I would like to thank my lovely parents, my dearest sister and my amazing friends, especially Pegah, for their unconditional love and support. I am very lucky to have these beautiful people during this journey. 


\section{Contents}

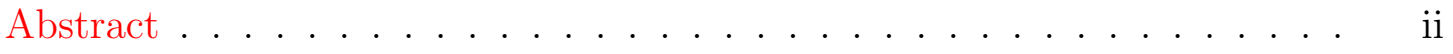

Aknowledgements ........................ iii

1 Introduction 1

2 Mathematical preliminaries $\quad 5$

2.1 Linear operators and matrices . . . . . . . . . . . . . 9

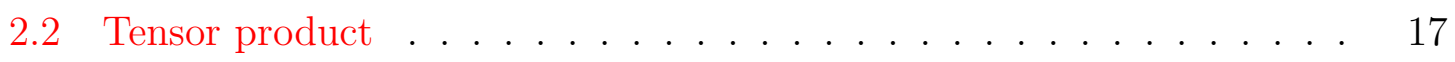

2.2.1 The operator-vector correspondence . . . . . . . . . . . 20

$\begin{array}{lll}3 & \text { Introduction to quantum information theory } & 21\end{array}$

3.1 The Pauli matrices . . . . . . . . . . . . . . . . . . . 22

3.1 .1 The Pauli group . . . . . . . . . . . . . . . . . 22

3.2 Quantum measurements . . . . . . . . . . . . . . . 25

3.3 Reduction, extension, and purification $\ldots \ldots \ldots \ldots$

3.4 Quantum channels . . . . . . . . . . . . . . . . . . 31

4 Classical error correction $\quad 39$

4.1 Error detection and error correction . . . . . . . . . . . . . 39

4.1 .1 Decoding . . . . . . . . . . . . . . . . 42 
5 Quantum error correction $\quad 45$

5.1 Quantum errors ....................... 45

5.2 Error correction: classical vs. quantum . . . . . . . . . . . . 47

5.3 Active methods of quantum error correction . . . . . . . . . . . 48

5.3.1 Mathematical formalism . . . . . . . . . . . . . 52

5.4 Passive methods of quantum error correction . . . . . . . . . . . 57

5.4.1 Decoherence-free subspaces . . . . . . . . . . . . 58

5.4 Noiseless subsystems .................. 60

5.5 Operator quantum error correction ............ 68

$\begin{array}{ll}\text { Bibliography } & 75\end{array}$ 


\section{Chapter 1}

\section{Introduction}

Postulates of the quantum mechanics promise the possibility of having substantially more effective computation devices. This was in fact manifested concretely in Shor's groundbreaking work [32]. A lot of attention has devoted to the subject ever since, and in fact, quantum computation and quantum information theories have become among the most active subjects in applied and theoretical mathematics and physics.

The main advantage of quantum devices over their classical counterpart is the possibility of taking advantage of quantum parallelism: the coherent interference pattern between the multitude of superpositions, which is the key feature in developing exponentially faster algorithm using quantum systems.

But, ironically, it is this very same feature of quantum systems that makes it also substantially more difficult to function (or even build) quantum devices! The fragility of a quantum state, which we can use to take advantage of the power of entanglement, also can result in decoherence: unwanted interference between the state of the quantum system carrying, or storing the information with the environment.

In fact, it has been clear since the birth of the theory that maintaining quantum coherence in any computing system is very essential in the attempt of exploiting 
the new possibilities opened up by applications of quantum mechanics in classical computations.

Of course any real-life device unavoidably interacts with its environment, and this can eventually lead to wrong outputs. In classical systems, this difficulty is dealt with using redundancy in encoding information. In fact the idea is very simple: transmit enough copies of the same information through the device that even after all the errors occurred, there will be still some copies unaffected.

When it comes to dealing with quantum information, unfortunately, such a simple redundancy scheme is not possible to use, primarily because the no-cloning theorem, that prevents the duplication of quantum states.

This is of course only the problem of how-to-encode the information. But, there is also a major obstacle in imitating classical techniques of error correction to deal with quantum information, and that is how-to-decode erupted state to restore the original information intended to be transmitted. This problem as well exists as a result of the nature of the quantum mechanics: to correct, or undo the effect of, the errors that have occurred during the transmition of the information, we of course need to detect the type of errors, and locate the part of information that has been corrupted. This is done simply by a suitable measurement in classical devices. But, what makes quantum systems fundamentally different from classical ones, is that measurement on a quantum system can completely destroy its state. Hence in our case, any attempt to detect the error, which is an essential part of any possible way of error correction may result in the complete lost of information.

In light of these obstacles, lots of doubts have been expressed about the feasibility of the large scale application of quantum computers. But it would be interesting to compare the situation of quantum computation today to that of classical computers in their early days. At that time it was often said that classical computers would not 
be very useful because errors in the computer itself would render the result untrustworthy. These doubts disappeared after the discovery of powerful error-correction techniques [41].

A breakthrough came to light when Shor [32] and Steane [35], independently showed that in a restricted model of errors (similar to what is usually assumed for classical error correction), it is possible to restore corrupted information by applying a redundancy-type scheme for encoding, and partial measurements for detecting errors.

This was the birth of the theory of quantum error correction: the subject of this thesis. Many codes have since been discovered to correct specific types of error, and a number of general techniques have been proposed to deal with large classes of error types. As a result, it may now be possible to implement practical quantum memories and achieve very reliable quantum communication.

This aim of this thesis is to provide a non-technical mathematical formalism for the theory of quantum error correction. We have tried to cover the main ideas of correcting schemes and techniques laid in a more mathematically formal framework without trapping the reader lost in a mountain of computational and physical technicalities.

The presentation of the concept in this format has not been appeared elsewhere, but we emphasize that none of the results presented in this thesis are my original work. All results have been collected from various work that have been cited in the list of references.

The thesis is organized as follows. Chapter 2 is devoted to a brief review of mathematical basics that we use in the rest of the thesis. This chapter is in fact intended to set our mathematical language and terminology rather than a thorough course on mathematical preliminaries.

Chapter 3 gives an introduction to the quantum information theory. Although we 
tried to introduce the main concepts of our concern with some details, again it is not intended to be a complete course or survey on the theory. We refer the reader to [28] for a more thorough treatment of the theory.

In Chapter 4 we give a brief introduction to the well-known classical techniques of error correction. We provide some examples to demonstrate the main ideas of such techniques and schemes. In this chapter, we intend to develop a motivation for the main content of this thesis, which appears in the last chapter.

Chapter 5 is the main part of this thesis, where we treat the theory of quantum error correction. After reviewing some concrete examples, we give the mathematical formalism of the main techniques of quantum error correcting. 


\section{Chapter 2}

\section{Mathematical preliminaries}

In this section we will review fundamentals of linear algebra. This materials can be found in any standard book on linear algebra. Our main references here are [37] and [26].

Recall that a vector space $V$ over a scalar field $F$ is an abelian group together with an associative scalar multiplication by elements of $F$. For any finite, nonempty set $\Sigma$, we denote by $\mathbb{C}^{\Sigma}$ the vector space of all functions from $\Sigma$ to the set of complex numbers $\mathbb{C}$, equipped with the pointwise addition and scalar multiplication. By a complex Euclidean space we mean a space of the form $\mathbb{C}^{\Sigma}$ for some finite, nonempty set $\Sigma$.

The elements of a vector space are called vectors. Vectors are sometimes denoted by using column matrix notations:

$$
\left(\begin{array}{c}
z_{1} \\
z_{2} \\
\vdots \\
z_{n}
\end{array}\right)
$$

The standard quantum mechanical notation for a vector in a vector space is $|\varphi\rangle$ 
( $\varphi$ is a label for a vector) and is called a ket.

Definition 2.0.1. A set $D$ in the Euclidean space $\mathbb{C}^{\Sigma}$ is convex if it contains line segments connecting any pair of its points. In other words $D \subseteq \mathbb{C}^{\Sigma}$ is convex if

$$
t x+(1-t) y \in D
$$

for all $x, y \in D$ and $t \in[0,1]$.

Definition 2.0.2. A function $f$ is convex over an interval $(a, b)$ if

$$
f\left(\gamma x_{1}+(1-\gamma) x_{2}\right) \leq \gamma f\left(x_{1}\right)+(1-\gamma) f\left(x_{2}\right) \quad \forall x_{1}, x_{2} \in(a, b) \quad \text { and } \quad 0 \leq \gamma \leq 1
$$

A convex function is strictly convex if equality holds only for $\gamma=0$ or $\gamma=1$.

A function $f$ is said to be concave over an interval $(a, b)$ if $-f$ is convex over the same interval.

Definition 2.0.3. An extreme point of a convex set $A$, is a point $x \in A$, with the property that if $x=t y+(1-t) z$ with $y, z \in A$ and $t \in[0,1]$, then $y=x$ and/or $z=x$. We denote by $\mathcal{E}(A)$ the set of extreme points of $A$.

An inner product on a vector space $V$ is a map $\langle\cdot \mid \cdot\rangle: V \times V \rightarrow \mathbb{C}$ that satisfies:

1. linearity in the second argument: $\langle u \mid \alpha v+\beta w\rangle=\alpha\langle u \mid v\rangle+\beta\langle u \mid w\rangle$, for all $|u\rangle,|v\rangle,|w\rangle \in V$ and $\alpha, \beta \in \mathbb{C}$

2. conjugate symmetry: $\langle v \mid u\rangle=\overline{\langle u \mid v\rangle}$;

3. positive definiteness: $\langle v \mid v\rangle \geq 0$ with equality if and only if $|v\rangle=0$.

A vector space equipped with an inner product is called an inner product space.

A spanning set of a vector space $V$ is a set of vectors $\left\{\left|v_{1}\right\rangle,\left|v_{2}\right\rangle, \ldots,\left|v_{n}\right\rangle\right\}$ in $V$ such that any vector $|v\rangle \in V$ can be written as a linear combination of these vectors: 


$$
|v\rangle=\sum_{i=1}^{i=n} a_{i}\left|v_{i}\right\rangle .
$$

A set of non-zero vectors $\left\{\left|v_{1}\right\rangle,\left|v_{2}\right\rangle, \ldots,\left|v_{n}\right\rangle\right\}$ is linearly dependent if there exists a set of complex numbers $a_{1}, a_{2}, \ldots, a_{n}$ with $a_{i} \neq 0$ for at least one value of $i$, such that

$$
a_{1}\left|v_{1}\right\rangle+a_{2}\left|v_{2}\right\rangle+\ldots+a_{n}\left|v_{n}\right\rangle=0 .
$$

A set of vectors which are not linearly dependent is linearly independent.

A linearly independent spanning subset of $V$ is called a basis for $V$.

Any two bases for $V$ have the same number of cardinality. The dimension of a vector space is the cardinality of a basis for $V$.

The dual of a vector space $V$, is the set of all scalar-valued linear maps $\varphi: V \rightarrow \mathbb{C}$ and is denoted by $V^{*}$. If we define addition and scalar multiplication in $V^{*}$ as

$$
(\varphi+\phi)(|v\rangle)=\varphi(|v\rangle)+\phi(|v\rangle), \text { and }(c \varphi)(|v\rangle)=c \varphi(|v\rangle)
$$

for $\varphi, \phi \in V^{*},|v\rangle \in V$ and $c \in \mathbb{C}$, then the dual is also a vector space over $\mathbb{C}$. If $V$ is an $n$-dimensional vector space, then so is $V^{*}$, and if $\left\{\left|b_{1}\right\rangle,\left|b_{2}\right\rangle, \ldots,\left|b_{n}\right\rangle\right\}$ is a basis for $V$, then the vectors $\left\{\left|b^{1}\right\rangle,\left|b^{2}\right\rangle, \ldots,\left|b^{n}\right\rangle\right\}$ defined by the property

$$
\left(\left|b^{i}\right\rangle,\left|b_{j}\right\rangle\right)=\delta_{i j}= \begin{cases}1 & \text { if } i=j \\ 0 & \text { if } i \neq j\end{cases}
$$

form a basis for $V^{*}$.

Vectors $|v\rangle$ and $|w\rangle$ are orthogonal if their inner product is zero, i.e. $\langle v \mid w\rangle=0$.

The Euclidean norm of a vector $|u\rangle \in \mathbb{C}^{\Sigma}$ is defined as

$$
\|u\|=\sqrt{\langle u \mid u\rangle}=\sqrt{\sum_{a \in \Sigma}|u(a)|^{2}} .
$$

The Euclidean norm satisfies the following properties: 
1. positive definiteness: $\|u\| \geq 0$, for all $|u\rangle \in \mathbb{C}^{\Sigma}$, with $\|u\|=0$ if and only if $u=0$.

2. $\|\alpha u\|=|\alpha|\|u\|, \quad \forall|u\rangle \in \mathbb{C}^{\Sigma}$ and $\alpha \in \mathbb{C}$.

3. The triangle inequality: $\|u+v\| \leq\|u\|+\|v\| \quad \forall|u\rangle,|v\rangle \in \mathbb{C}^{\Sigma}$.

The above properties are the defining properties of any function that is called a norm.

For $1 \leq p<\infty$, the $p$-norm is defined by

$$
\|u\|_{p}=\left(\sum_{a \in \Sigma}|u(a)|^{p}\right)^{1 / p} \text { for each }|u\rangle \in \mathbb{C}^{\Sigma}
$$

and

$$
\|u\|_{\infty}=\max \{|u(a)|: a \in \Sigma\} .
$$

The Euclidean norm corresponds to the case $p=2$.

A unit vector is a vector whose norm is 1 . We also say that $|v\rangle$ is normalized if $\||v\rangle \|=1$. So in order to normalize a vector it is enough to divide it by its norm, $|v\rangle / \||v\rangle \|$

A set $\left\{\left|v_{1}\right\rangle,\left|v_{2}\right\rangle, \ldots,\left|v_{n}\right\rangle\right\}$ of vectors is orthonormal if $\left\langle v_{i} \mid v_{j}\right\rangle=\delta_{i j}, \forall i, j \in\{1,2, \ldots, n\}$.

The Cauchy-Schwarz inequality states that

$$
|\langle u, v\rangle| \leq\|u\|\|v\| \forall u, v \in \mathbb{C}^{\Sigma},
$$

and the equality occurs if and only if $|u\rangle$ and $|v\rangle$ are linearly dependent, i.e. $|u\rangle=\alpha|v\rangle$ for some $\alpha \in \mathbb{C}$. 


\subsection{Linear operators and matrices}

Definition 2.1.1. A linear operator between two vector spaces $\mathcal{X}$ and $\mathcal{Y}$ is a mapping $A: \mathcal{X} \rightarrow \mathcal{Y}$ which satisfies

$$
A(a|v\rangle+|w\rangle)=a A|v\rangle+A|w\rangle, \quad \forall a \in \mathbb{C},|v\rangle,|w\rangle \in \mathcal{X}
$$

The collection of all linear operators from $\mathcal{X}$ to $\mathcal{Y}$ is represented by $L(\mathcal{X}, \mathcal{Y})$. A linear transformation is a linear operator, with $\mathcal{X}=\mathcal{Y}$. The identity operator $I: \mathcal{X} \rightarrow \mathcal{X}$, maps $|\varphi\rangle$ to itself, $I|\varphi\rangle=|\varphi\rangle$.

The set $L(\mathcal{X}, \mathcal{Y})$ is a vector space when addition and scalar multiplication is defined in the following way:

1. Addition: $\forall A, B \in L(\mathcal{X}, \mathcal{Y})$, the operator $A+B \in L(\mathcal{X}, \mathcal{Y})$ is defined by the following equation:

$$
(A+B)|v\rangle=A|v\rangle+B|v\rangle
$$

$\forall|v\rangle \in \mathcal{X}$

2. Scalar multiplication: $\forall A \in L(\mathcal{X}, \mathcal{Y})$ and $\alpha \in \mathbb{C}$, the operator $\alpha A \in L(\mathcal{X}, \mathcal{Y})$ is defined by the equation:

$$
(\alpha A)|v\rangle=\alpha A|v\rangle
$$

$\forall|v\rangle \in \mathcal{X}$

And the dimension of this vector space is

$$
\operatorname{dim}(L(\mathcal{X}, \mathcal{Y}))=\operatorname{dim}(\mathcal{X}) \operatorname{dim}(\mathcal{Y})
$$


Linear operators from one complex Euclidean space $\mathcal{V}$ to another complex Euclidean space $\mathcal{W}$ are naturally represented by matrices. Suppose $\left\{\left|b_{1}\right\rangle,\left|b_{2}\right\rangle, \ldots,\left|b_{n}\right\rangle\right\}$ and $\left\{\left|b_{1}^{\prime}\right\rangle,\left|b_{2}^{\prime}\right\rangle, \ldots,\left|b_{m}^{\prime}\right\rangle\right\}$ are bases for $\mathcal{V}$ and $\mathcal{W}$ respectively, then a linear transformation $A$ is represented by an $m \times n$ matrix, $\left[a_{i j}\right]$, in a way that if

$$
|v\rangle=\sum_{i=1}^{n} v_{i}\left|b_{i}\right\rangle \text { and }|w\rangle=\sum_{j=1}^{m} w_{k}\left|b_{k}^{\prime}\right\rangle,
$$

and $|w\rangle=A|v\rangle$, then $w_{k}=\sum_{j=1}^{n} a_{k j} v_{j}$, which can be written as

$$
\left(\begin{array}{c}
w_{1} \\
w_{2} \\
\vdots \\
w_{m}
\end{array}\right)=\left(\begin{array}{cccc}
a_{11} & a_{12} & \cdots & a_{1 n} \\
a_{21} & a_{22} & \cdots & a_{2 n} \\
\vdots & \vdots & \vdots & \vdots \\
a_{m 1} & a_{m 2} & \cdots & a_{m n}
\end{array}\right)\left(\begin{array}{c}
v_{1} \\
v_{2} \\
\vdots \\
v_{n}
\end{array}\right) .
$$

The kernel of an operator $A \in L(\mathcal{X}, \mathcal{Y})$ is defined as

$$
\operatorname{ker}(A)=\{u \in \mathcal{X}: A u=0\},
$$

and it is a subspace of $\mathcal{X}$. The image of an operator is defined as

$$
\operatorname{Im}(A)=\{A u: u \in \mathcal{X}\}
$$

which is also a subspace of $\mathcal{Y}$.

Rank of $A$ is dimension of $\operatorname{Im}(A)$. For every operator $A \in L(\mathcal{X}, \mathcal{Y})$ the following is true,

$$
\operatorname{dim}(\mathcal{X})=\operatorname{rank}(A)+\operatorname{dim}(\operatorname{ker}(A))
$$

An eigenvector, $|v\rangle$, of a linear transformation $A$ is a nonzero vector such that

$$
A|v\rangle=\lambda|v\rangle
$$


for some $\lambda \in \mathbb{C}$. The scalar, $\lambda$, is called the eigenvalue corresponding to the eigenvector $|v\rangle$ of $A$.

A diagonal representation for an operator $A$ on a vector space $V$ is a representation $A=\sum_{i} \lambda_{i}|i\rangle\langle i|$, where vector $|i\rangle$ form an orthonormal set of eigenvectors for $A$, with corresponding eigenvalues $\lambda_{i}$. An operator is said to be diagonalizable if it has a diagonal representation.

Definition 2.1.2. The trace of a square matrix $A=\left[a_{i j}\right]$, is the sum of the elements on the main diagonal of the matrix:

$$
\operatorname{tr}(A)=a_{11}+a_{22}+\ldots+a_{n n}=\sum_{i=1}^{n} a_{i i} .
$$

The trace has the following properties:

1. the trace is a linear map: given a scalar $\alpha \in \mathbb{C}$, then $\operatorname{tr}(\alpha A+B)=\alpha \operatorname{tr}(A)+$ $\operatorname{tr}(B)$

2. the trace is not affected by the order of the matrices in a product of two matrices: $\operatorname{tr}(A B)=\operatorname{tr}(B A)$

3. $\operatorname{tr}\left(A^{*} A\right) \geq 0$ and $\operatorname{tr}\left(A^{*} A\right)=0 \Rightarrow A=0$.

We define the trace of an operator $A$ to be the trace of any matrix representation of $A$. Note that by property (2) above, this is well-defined. Let $\mathcal{X}$ and $\mathcal{Y}$ be arbitrary complex Euclidean spaces and $A, B$ be linear operators in $L(\mathcal{X}, \mathcal{Y})$. Using the trace we can define an inner product on the space $L(\mathcal{X}, \mathcal{Y})$, by

$$
\langle A, B\rangle=\operatorname{tr}\left(A^{*} B\right) \quad \forall A, B \in L(\mathcal{X}, \mathcal{Y})
$$

This inner product is known as Hilbert-Schmidt or trace inner product.

An $n$-dimensional Hilbert space, $\mathcal{H}_{n}$, is an $n$-dimensional vector space over the field of complex numbers, $\mathbb{C}$, equipped with an inner product. An example of a Hilbert space of dimension $n$ is $\mathbb{C}^{n}$ when equipped with the inner product: 


$$
\left(\left(y_{1}, y_{2}, \ldots, y_{n}\right),\left(z_{1}, z_{2}, \ldots, z_{n}\right)\right) \equiv \sum_{i} \bar{y}_{i} z_{i}=\left(\bar{y}_{1}, \bar{y}_{2}, \ldots, \bar{y}_{n}\right)\left(\begin{array}{c}
z_{1} \\
z_{2} \\
\vdots \\
z_{n}
\end{array}\right)
$$

where $\bar{y}_{i}$ 's are complex conjugate of $y_{i}$ 's. This Hilbert space has a canonical orthonormal basis:

$$
(1,0, \ldots, 0),(0,1, \ldots, 0), \ldots,(0,0, \ldots, 1)
$$

The canonical orthonormal basis of $\mathbb{C}^{n}$ can be expressed as ket vectors as

$$
\{|0\rangle,|1\rangle, \ldots,|n-1\rangle\}
$$

or, as bra vectors as

$$
\{\langle 0|,\langle 1|, \ldots,\langle n-1|\} \text {. }
$$

We will also use the matrix representation of each ket vector $|i\rangle$ in this canonical representation as a column vector is

$$
|0\rangle=\left(\begin{array}{c}
1 \\
0 \\
\vdots \\
0 \\
\vdots \\
0
\end{array}\right),|1\rangle=\left(\begin{array}{c}
0 \\
1 \\
\vdots \\
0 \\
\vdots \\
0
\end{array}\right), \ldots,|i\rangle=\left(\begin{array}{c}
0 \\
0 \\
\vdots \\
1 \\
\vdots \\
0
\end{array}\right), \ldots,|n-1\rangle=\left(\begin{array}{c}
0 \\
0 \\
\vdots \\
0 \\
\vdots \\
1
\end{array}\right),
$$

and the matrix representation of the bra vector $\langle i|$ as a row vector is

$$
\begin{gathered}
\langle 0|=\left(\begin{array}{cccccc}
1 & 0 & \ldots & 0 & \ldots & 0
\end{array}\right), \\
\langle 1|=\left(\begin{array}{llllll}
0 & 1 & \ldots & 0 & \ldots & 0
\end{array}\right), \\
\vdots
\end{gathered}
$$




$$
\begin{aligned}
& \langle i|=\left(\begin{array}{llllll}
0 & 0 & \ldots & 1 & \ldots & 0
\end{array}\right), \\
& \langle n-1|=\left(\begin{array}{llllll}
0 & 0 & \ldots & 0 & \ldots & 1
\end{array}\right) .
\end{aligned}
$$

So, an $n$-dimensional ket $|u\rangle$ can be expressed as a linear combination of the orthonormal basis ket vectors

$$
|u\rangle=a_{0}|0\rangle+a_{1}|1\rangle+\ldots+a_{i}|i\rangle+\ldots+a_{n-1}\left|v_{n-1}\right\rangle,
$$

where $a_{0}, a_{1}, \ldots, a_{i}, \ldots, a_{n-1}$ are complex numbers. for each ket vector $|u\rangle$ there is a dual, the bra vector denoted by $\langle u|$. The bra is the Hermitian conjugation of the ket and vice versa.

$$
\langle u|=\bar{a}_{0}\langle 0|+\bar{a}_{1}\langle 1|+\ldots+\bar{a}_{i}\langle i|+\ldots+\bar{a}_{n-1}\left\langle v_{n-1}\right| .
$$

In matrix representation, a ket vector is expressed as the column matrix

$$
|u\rangle=\left(\begin{array}{c}
a_{0} \\
a_{1} \\
\vdots \\
a_{i} \\
\vdots \\
a_{n-1}
\end{array}\right) .
$$

And a dual bra vector is expressed as the row matrix

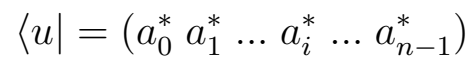

Suppose $A$ is any linear operator on a Hilbert space $\mathcal{H}_{n}$. There exists a unique linear operator $A^{*}$ on $\mathcal{H}_{n}$ such that for all vectors $|u\rangle,|v\rangle \in \mathcal{H}_{n}$,

$$
\langle u \mid A v\rangle=\left\langle A^{*} u \mid v\right\rangle
$$


This linear operator is known as the adjoint or Hermitian conjugate of the operator $A$. From the above definition it is easy to see $(A B)^{*}=B^{*} A^{*}$.

If $A$ is represented by the matrix $A=\left[a_{i j}\right]$, then the matrix of the adjoint $A^{*}$ of $A$ is the transpose conjugate matrix of $A$, that is

$$
A^{*}=\left[a_{j i}^{*}\right], 1 \leq i, j \leq n
$$

Definition 2.1.3. An operator $A$ is normal if $A^{*} A=A A^{*}$. An operator $A$ is Hermitian (self-adjoint) if $A=A^{*}$.

A hermitian operator is normal. The sum of two Hermitian operators is Hermitian, but their product is not necessarily Hermitian.

A projection is a Hermitian operator $P$ such that $P^{2}=P$. The outer product $|\varphi\rangle\langle\varphi|$ of a unit vector $|\varphi\rangle$ with itself, is the projection

$$
P_{\varphi}|\psi\rangle=(\langle\varphi \mid \psi\rangle)|\varphi\rangle
$$

Two projections, $P_{i}$ and $P_{j}$, are orthogonal if $P_{i} P_{j}|\varphi\rangle=0$ for every unit vector $\varphi \in \mathcal{H}_{n}$. A set of orthogonal projections, $\left\{P_{1}, P_{2}, \ldots\right\}$ is complete if

$$
\sum_{i} P_{i}=I
$$

A matrix $U$ is said to be unitary if $U U^{*}=U^{*} U=I$. An operator on a Hilbert space is unitary if each of its matrix representation is unitary. The product of two unitary operators is unitary, but the sum is not. A unitary operator $A$ preserve the inner product between vectors:

$$
\langle U v \mid U w\rangle=\langle v \mid w\rangle .
$$

Another special subclass of Hermitian operators are positive operators.

An operator $A: \mathcal{H}_{n} \rightarrow \mathcal{H}_{n}$ is positive definite if 


$$
\langle A \varphi \mid \varphi\rangle>0, \quad \forall|\varphi\rangle \in \mathcal{H}_{n}, \quad|\varphi\rangle \neq 0 .
$$

The eigenvalues of a positive definite operator are real and positive.

An operator $A$ is positive semi-definite if

$$
\langle A \varphi \mid \varphi\rangle \geq 0, \quad \forall|\varphi\rangle \in \mathcal{H}_{n}, \quad|\varphi\rangle \neq 0 .
$$

A positive semi-definite operator has real and non-negative eigenvalues; thus $\operatorname{tr}(A) \geq$ 0. A positive semi-definite operator is self-adjoint. In fact positive definite operators are positive semidefinite operators which are invertible. For a given complex Euclidean space $\mathcal{X}, \operatorname{Pd}(\mathcal{X})$ and $\operatorname{Pos}(\mathcal{X})$ denote positive definite and positive semidefinite operators on $\mathcal{X}$, respectively.

Definition 2.1.4. Density operators are positive semi-definite operators having trace equal to 1 . For a given complex Euclidean space $\mathcal{X}$, the notation

$$
D(\mathcal{X})=\{\rho \in \operatorname{Pos}(\mathcal{X}): \operatorname{tr}(\rho)=1\}
$$

is used to denote the collection of density operators acting on $\mathcal{X}$.

Among all of the norms on the space of operators of particular interest for us is the class of Schatten p-norm (defined below). It includes the spectral norm, the Frobenius norm and the trace norm (all introduced below) which are the most commonly used norms in quantum information theory.

Let $A \in L(\mathcal{X}, \mathcal{Y})$ be an operator; the Schatten p-norm of A is

$$
\|A\|_{p}=\left[\operatorname{tr}\left(A^{*} A\right)^{p / 2}\right]^{1 / p},
$$

for $p \geq 1$ and

$$
\|A\|_{\infty}=\sup \{\|A u\|: u \in \mathcal{X},\|u\|=1\} .
$$

The Schatten p-norms satisfy the following properties: 
1. For any operator $A \in L(\mathcal{X}, \mathcal{Y})$,

$$
\|A\|_{p}=\left\|A^{*}\right\|_{p} .
$$

2. For any operator $A \in L(\mathcal{X}, \mathcal{Y})$ and $1 \leq p \leq q \leq \infty$ we have $\|A\|_{p} \geq\|A\|_{q}$.

3. The Schatten norms satisfy a type of Hölder inequality:

$$
|\langle B, A\rangle| \leq\|A\|_{p}\|B\|_{q}, 1 / p+1 / q=1,
$$

which follows from

$$
\|A\|_{p}=\max \left\{|\langle B, A\rangle|: B \in L(\mathcal{X}, \mathcal{Y}),\|B\|_{q} \leq 1\right\} .
$$

4. The Schatten p-norm is isometrically and unitarily invariant for any $p \in[1, \infty]$. This means that for any choice of linear isometries $U$ and $V$ (which include unitary operators $U$ and $V$ ) for which the product $U A V^{*}$ makes sense we have

$$
\|A\|_{p}=\left\|U A V^{*}\right\|_{p}
$$

5. For any choice of linear operators $A \in L\left(\mathcal{X}_{1}, \mathcal{X}_{2}\right), B \in L\left(\mathcal{X}_{2}, \mathcal{X}_{3}\right)$, and $C \in$ $L\left(\mathcal{X}_{3}, \mathcal{X}_{4}\right)$, and any choice of $p \in[1, \infty]$, it satisfies

$$
\|C B A\|_{p} \leq\|C\|_{\infty}\|B\|_{p}\|A\|_{\infty} .
$$

6. For any $A, B \in L(\mathcal{X}),\|A B\|_{p} \leq\|A\|_{p}\|B\|_{p}$.

Remark 2.1.1. - The Schatten 1-norm, also called the trace norm, is sometimes denoted by $\|\cdot\|_{t r}$, and satisfies

$$
\|A\|_{1}=\sup \{|\langle A, U\rangle|: U \in L(\mathcal{X}) \text { is unitary }\}
$$


- The Schatten 2-norm, also called the Frobenius norm, is sometimes denoted by $\|\cdot\|_{F}$. It is the norm defined by the inner product on $L(\mathcal{X})$ :

$$
\|A\|_{2}=\sqrt{\langle A, A\rangle}
$$

- The Schatten $\infty$-norm also called the spectral norm, or the operator norm, and is ofte denoted by $\|\cdot\|$. It satisfies

$$
\left\|A^{*} A\right\|=\|A\|^{2} .
$$

We conclude this section with a fundamental decomposition theorem for normal linear operators.

Theorem 2.1.1 (The spectral Theorem). Let $\mathcal{X}$ be a complex Euclidean space and $A \in L(\mathcal{X})$. Then $A$ is normal if and only if there exists an orthonormal basis for $\mathcal{X}$ consisting of eigenvectors for A.

A useful corollary of the spectral theorem is:

Corollary 2.1.1. [37, Corollary 1.4] Suppose $\mathcal{X}$ is a complex Euclidean space. Let $A \in L(\mathcal{X})$ be a normal operator and $\lambda_{1}, \lambda_{2}, \ldots, \lambda_{n}$ are the eigenvalues of $A$. Then there exist an orthonormal basis $\left\{x_{1}, x_{2}, \ldots, x_{n}\right\}$ of $\mathcal{X}$ such that

$$
A=\sum_{i=1}^{n} \lambda_{i} x_{i} x_{i}^{*}
$$

\subsection{Tensor product}

Suppose $\mathcal{V}$ and $\mathcal{W}$ are $m$ and $n$-dimensional Hilbert spaces, with bases $\left\{\left|v_{1}\right\rangle,\left|v_{2}\right\rangle, \ldots,\left|v_{m}\right\rangle\right\}$ and $\left\{\left|w_{1}\right\rangle,\left|w_{2}\right\rangle, \ldots,\left|w_{n}\right\rangle\right\}$, respectively. Their tensor product is the (abstract) mndimensional vector space $\mathcal{V} \otimes \mathcal{W}$ with the basis $\left\{\left|v_{i}\right\rangle \otimes\left|w_{j}\right\rangle: 1 \leq i \leq m, 1 \leq j \leq n\right\}$; that is $\mathcal{V} \otimes \mathcal{W}$ consists of formal linear combinations of the elements of the basis 
$\left\{\left|v_{i}\right\rangle \otimes\left|w_{j}\right\rangle: 1 \leq i \leq m, 1 \leq j \leq n\right\}$. We can define $|v\rangle \otimes|w\rangle$, the tensor product of any two elements $|v\rangle \in \mathcal{V}$ and $|w\rangle \in \mathcal{W}$, by declaring the following properties:

1. For an arbitrary scalar $c$ and elements $|v\rangle \in \mathcal{V}$ and $|w\rangle \in \mathcal{W}$,

$$
c(|v\rangle \otimes|w\rangle)=(c|v\rangle) \otimes|w\rangle=|v\rangle \otimes(c|w\rangle) .
$$

2. For arbitrary $|v\rangle,\left|v^{\prime}\right\rangle \in \mathcal{V}$ and $|w\rangle \in \mathcal{W}$,

$$
\left(|v\rangle+\left|v^{\prime}\right\rangle\right) \otimes|w\rangle=|v\rangle \otimes|w\rangle+\left|v^{\prime}\right\rangle \otimes|w\rangle .
$$

3. For arbitrary $|v\rangle \in \mathcal{V}$ and $|w\rangle,\left|w^{\prime}\right\rangle \in \mathcal{W}$,

$$
|v\rangle \otimes\left(|w\rangle+\left|w^{\prime}\right\rangle\right)=|v\rangle \otimes|w\rangle+|v\rangle \otimes\left|w^{\prime}\right\rangle
$$

We next define tensor product of linear operators. Let $A$ and $B$ are linear operator on $\mathcal{V}$ and $\mathcal{W}$, respectively. We define the linear operator $A \otimes B$ on $\mathcal{V} \otimes \mathcal{W}$ by the equation

$$
(A \otimes B)\left(\left|v_{i}\right\rangle \otimes\left|w_{j}\right\rangle\right):=A\left|v_{i}\right\rangle \otimes B\left|w_{j}\right\rangle \quad(1 \leq i \leq m, 1 \leq j \leq n) .
$$

An arbitrary linear operator $C: \mathcal{V} \otimes \mathcal{W} \rightarrow \mathcal{V}^{\prime} \otimes \mathcal{W}^{\prime}$ can be represented as a linear combination of tensor product of operators mapping $\mathcal{V}$ to $\mathcal{V}^{\prime}$ and $\mathcal{W}$ to $\mathcal{W}^{\prime}$,

$$
C=\sum_{i} c_{i} A_{i} \otimes B_{i}
$$

where by definition

$$
\sum_{i} c_{i} A_{i} \otimes B_{i}(|v\rangle \otimes|w\rangle)=\sum_{i} c_{i} A_{i}|v\rangle \otimes B_{i}|w\rangle .
$$

The inner product on the spaces $\mathcal{V}$ and $\mathcal{W}$ can be used to define an inner product on $\mathcal{V} \otimes \mathcal{W}$ 


$$
\left.\left\langle\sum_{i} a_{i} \mid u_{i}\right\rangle \otimes\left|z_{i}\right\rangle, \sum_{j} b_{j}\left|u_{j}^{\prime}\right\rangle \otimes\left|z_{j}^{\prime}\right\rangle\right\rangle=\sum_{i j} \bar{a}_{i} b_{j}\left\langle u_{i} \mid u_{j}^{\prime}\right\rangle\left\langle z_{i} \mid z_{j}^{\prime}\right\rangle .
$$

If $A$ and $B$ are two linear operators in $L\left(\mathcal{X}_{1}, \mathcal{X}_{2}\right)$ and $L\left(\mathcal{Y}_{1}, \mathcal{Y}_{2}\right)$, respectively, and $\mathcal{X}_{1}, \mathcal{X}_{2}, \mathcal{Y}_{1}$ and $\mathcal{Y}_{2}$ are complex Euclidean spaces, then

$$
(A \otimes B)^{*}=A^{*} \otimes B^{*}
$$

This implies if $A$ and $B$ are Hermitian, then so is $A \otimes B$.

If $A$ and $B$ are two linear operators represented by $m \times n$ and $p \times q$ matrices:

$$
A=\left(\begin{array}{cccc}
a_{11} & a_{12} & \cdots & a_{1 n} \\
a_{21} & a_{22} & \cdots & a_{2 n} \\
\vdots & \vdots & \vdots & \vdots \\
a_{n 1} & a_{n 2} & \cdots & a_{m n}
\end{array}\right) \text { and } B=\left(\begin{array}{cccc}
b_{11} & b_{12} & \cdots & b_{1 q} \\
b_{21} & b_{22} & \cdots & b_{2 q} \\
\vdots & \vdots & \vdots & \vdots \\
b_{p 1} & b_{p 2} & \cdots & b_{p q}
\end{array}\right)
$$

then their tensor product $A \otimes B$ is represented (with respect to the canonical bases) by the $m p \times n q$ matrix

$$
A \otimes B=\left(\begin{array}{cccc}
a_{11} B & a_{12} B & \cdots & a_{1 n} B \\
a_{21} B & a_{22} B & \cdots & a_{2 n} B \\
\vdots & \vdots & \vdots & \vdots \\
a_{n 1} B & a_{n 2} B & \cdots & a_{m n} B
\end{array}\right) .
$$

Here, $a_{i j} B, 1 \leq i \leq m$, and $1 \leq j \leq n$, is a sub matrix whose entries are the products of $a_{i j}$ and all the elements of matrix $B$.

Let $\mathcal{X}$ and $\mathcal{Y}$ be complex Euclidean spaces. We represent trace as a mapping in the form $\operatorname{tr}: L(\mathcal{X}) \rightarrow L(\mathbb{C})$, by making the identification $L(\mathbb{C})=\mathbb{C}$. For the complex Euclidean space $\mathcal{Y}$ we define the partial trace by

$$
\operatorname{tr} \mathcal{Y}:=\operatorname{tr} \otimes 1_{L(\mathcal{Y})}: L(\mathcal{X} \otimes \mathcal{Y}) \rightarrow L(\mathcal{Y})
$$

which is the unique mapping that satisfies

$$
\operatorname{tr}_{\mathcal{Y}}(A \otimes B)=\operatorname{tr}(A) B ; \quad \forall A \in L(\mathcal{X}) \quad \text { and } B \in L(\mathcal{Y})
$$




\subsubsection{The operator-vector correspondence}

For given complex Euclidean spaces $\mathcal{X}$ and $\mathcal{Y}$, we can canonically identify the spaces $L(\mathcal{X}, \mathcal{Y})$ and $\mathcal{Y} \otimes \mathcal{X}$, via the vec map, which is defined as follows:

$$
\text { vec }: L(\mathcal{X}, \mathcal{Y}) \rightarrow \mathcal{Y} \otimes \mathcal{X} \quad E_{b, a} \mapsto e_{b} \otimes e_{a}
$$

The vec map is a linear bijection, which implies that every vector $u \in \mathcal{Y} \otimes \mathcal{X}$ uniquely determines an operator $A \in L(\mathcal{X}, \mathcal{Y})$ that satisfies $\operatorname{vec}(A)=u$. It is also an isometry, in the sense that

$$
\langle A, B\rangle=\langle\operatorname{vec}(A), \operatorname{vec}(B)\rangle
$$

for all $A, B \in L(\mathcal{X}, \mathcal{Y})$. The following properties of the vec map can be verified through straightforward calculations:

1. For $u \in \mathcal{X}$ and $v \in \mathcal{Y}$ we have

$$
\operatorname{vec}\left(u v^{*}\right)=u \otimes v
$$

2. For every choice of complex Euclidean spaces $\mathcal{X}_{1}, \mathcal{X}_{2}, \mathcal{Y}_{1}$, and $\mathcal{Y}_{2}$, and every choice of operators $A \in L\left(\mathcal{X}_{1}, \mathcal{Y}_{1}\right), B \in L\left(\mathcal{X}_{2}, \mathcal{Y}_{2}\right)$, and $X \in L\left(\mathcal{X}_{2}, \mathcal{X}_{1}\right)$, we have

$$
(A \otimes B) \operatorname{vec}(X)=\operatorname{vec}\left(A X B^{T}\right)
$$

3. For every choice of complex Euclidean spaces $\mathcal{X}$ and $\mathcal{Y}$, and every choice of operators $A, B \in L(\mathcal{X}, \mathcal{Y})$, the following equations hold:

$$
\begin{gathered}
\operatorname{tr}_{\mathcal{X}}\left(\operatorname{vec}(A), \operatorname{vec}\left(B^{*}\right)\right)=A B^{*}, \\
\operatorname{tr}_{\mathcal{Y}}\left(\operatorname{vec}(A) \operatorname{vec}(B)^{*}\right)=\left(B^{*} A\right)^{T} .
\end{gathered}
$$




\section{Chapter 3}

\section{Introduction to quantum information theory}

These materials presented in this chapter are mainly taken from [37] and [26]. A quantum system is an abstract device that stores quantum information. A quantum system $X$, is described in a finite dimensional Hilbert space $\mathcal{H}_{n}$, which is also called the state space of the system.

A qubit is the simplest quantum system, with a two-dimensional state space. Suppose $|0\rangle$ and $|1\rangle$ form an orthonormal basis for that state space. A qubit is a vector in the state space of the form

$$
|\varphi\rangle=\alpha|0\rangle+\beta|1\rangle, \quad \alpha, \beta \in \mathbb{C} .
$$

A quantum state is a unit vector $|\varphi\rangle$ (called the state vector) in a Hilbert space $\mathcal{H}_{n}$. The quantum information can be interpreted as information that is held in the state of a quantum system. 


\subsection{The Pauli matrices}

The so called Pauli matrices are among the most important operations in quantum information. Recall the Pauli matrices are defined as

$$
\begin{gathered}
\sigma_{0} \equiv I \equiv\left[\begin{array}{cc}
1 & 0 \\
0 & 1
\end{array}\right] ; \quad \sigma_{1} \equiv \sigma_{x} \equiv X \equiv\left[\begin{array}{cc}
0 & 1 \\
1 & 0
\end{array}\right] ; \\
\sigma_{2} \equiv \sigma_{y} \equiv Y \equiv\left[\begin{array}{cc}
0 & -i \\
i & 0
\end{array}\right] ; \quad \sigma_{3} \equiv \sigma_{z} \equiv Z \equiv\left[\begin{array}{cc}
1 & 0 \\
0 & 1
\end{array}\right] .
\end{gathered}
$$

\subsubsection{The Pauli group}

We first briefly introduce a few notion from group theory that we will use in later chapters.

Definition 3.1.1. A group is a set $G$ with an associative binary operation $(a, b) \mapsto a b$ satisfying the following properties:

1. there is an element $1 \in G$ such that $a 1=1 a=a$ for all $a$ in $G$,

2. each element $a \in G$ has an inverse, i.e. if $a$ is in $G$, then there is an element $a^{-1}$ in $G$ such that $a a^{-1}=a^{-1} a=1$.

A group $G$ is abelian if the binary operation is commutative, i.e. $a b=b a$ for all $a, b$ in $G$.

A subgroup $H$ of a group $G$ is a nonempty subset of $G$ that forms a group under the binary operation of $G$; or equivalently, a nonempty subset of $G$ such that if $a$ and $b$ belong to $H$, so does $a b^{-1}$. (Note that $1=a a^{-1} \in H$; also, $a b=a\left(\left(b^{-1}\right)^{-1}\right) \in H$.)

Suppose $G$ and $H$ are groups. A mapping $\varphi: G \rightarrow H$ is a homomorphism if $\varphi(g h)=\varphi(g) \varphi(h)$ for all $g, h \in G$. An isomorphism is a bijective homomorphism. If an isomorphism exists between two groups, we say that the groups are isomorphic. 
By an ( $n$-dimensional) representation of a group $G$ we mean a homomorphism $\pi: G \rightarrow G L(n)$, where $G L(n)$ denotes the groups of invertible $n \times n$ complex matrices.

Suppose $\pi_{1}$ and $\pi_{2}$ are, respectively, $m$ and $n$-dimensional representations of a group $G$. The direct sum of $\pi_{1}$ and $\pi_{2}$ is the $m+n$-dimensional representation $\pi_{1} \oplus \pi_{2}$ of $G$ defined by

$$
\pi_{1} \oplus \pi_{2}(g)\left(v_{1}, \ldots, v_{m}, v_{m+1}, \ldots, v_{m+n}\right)=\left(\pi_{1}(g)\left(v_{1}, \ldots, v_{m}\right), \pi_{2}(g)\left(v_{m+1}, \ldots, v_{m+n}\right)\right) .
$$

If $\pi=\pi_{1} \oplus \pi_{2}$, we say $\pi_{1}$ and $\pi_{2}$ are sub representations of $\pi$. A representation $\pi$ is said to be irreducible if $\pi=\pi_{1} \oplus \pi_{2}$ implies $\pi_{1}$ or $\pi_{2}$ is 0 -dimensional. Any (finite-dimensional) representation can be decomposed into a direct sum of irreducible representations.

The tensor product of $\pi_{1}$ and $\pi_{2}$ is the $m n$-dimensional representation $\pi_{1} \otimes \pi_{2}$ of $G$ defined by

$$
\pi_{1} \otimes \pi_{2}(g)=\pi_{1}(g) \otimes \pi_{2}(g)
$$

The four Pauli operators, $I, X, Z, Y$ allow us to express the four possible effects of the environment on a qubit in state $|\varphi\rangle=\alpha|0\rangle+\beta|1\rangle$ : no error (the qubit is unchanged), bit-flip, phase-flip, and bit- and phase-flip: 


$$
\begin{array}{ccc}
\text { No Error } & I=\left(\begin{array}{cc}
1 & 0 \\
0 & 1
\end{array}\right) & |\varphi\rangle=I|\varphi\rangle=\alpha_{0}|0\rangle+\alpha_{1}|1\rangle ; \\
\text { Bit-flip } & X=\left(\begin{array}{cc}
0 & 1 \\
1 & 0
\end{array}\right) & |\varphi\rangle=|\varphi\rangle=\alpha_{1}|0\rangle+\alpha_{0}|1\rangle ; \\
\text { Phase-flip } & Z=\left(\begin{array}{cc}
1 & 0 \\
0 & -1
\end{array}\right) & |\varphi\rangle=Z|\varphi\rangle=\alpha_{0}|0\rangle-\alpha_{1}|1\rangle ; \\
\text { Bit and phase-flip } & Y=i X Z=\left(\begin{array}{cc}
0 & -i \\
i & 0
\end{array}\right) & |\varphi\rangle=Y|\varphi\rangle=-i\left(\alpha_{1}|0\rangle-\alpha_{0}|1\rangle\right) .
\end{array}
$$

Pauli operators, $I, X, Y$, and $Z$ form a group and have several nice properties:

1. They anticommute:

$$
X Z=-Z X, \quad X Y=-Y X, \quad Y Z=-Z Y .
$$

2. The square of each is equal to $I$; this means that the transformation they perform on a qubit are reversible.

3. They span the space of $2 \times 2$ matrices.

The 1-qubit Pauli group $\mathcal{P}_{1}$ consists of the Pauli operators, $I, X, Y$, and $Z$ together with the multiplicative factors \pm 1 and $\pm i$

$$
\mathcal{P}_{1} \equiv\{ \pm I, \pm i I, \pm X, \pm i X, \pm Y, \pm i Y, \pm Z, \pm i Z\}
$$

The cardinality of $\mathcal{P}_{1}$ is $\left|\mathcal{P}_{1}\right|=16=2^{4}$.

Proposition 3.1.1. [26, Section 5.11] The members of the 1-qubit Pauli group $\mathcal{P}_{1}$ 
1. are unitary;

2. either commute or anticommute;

3. are either Hermitian, or anti-Hermitian.

Proof. Clearly all members are unitary because the four Pauli operators are multiplied with \pm 1 or $\pm i . X, Y, Z$ anticommute but $I$ commutes with each of them. $I, X, Y$ and $Z$ are Hermitian, but

$$
(i I)^{*}=-i I
$$

The joint state of two or more quantum systems with Hilbert spaces $\mathcal{H}_{n_{1}}, \mathcal{H}_{n_{2}}, \ldots, \mathcal{H}_{n_{k}}$ is a vector in the tensor product Hilbert space

$$
\mathcal{H}_{n_{1} \times n_{2} \times \ldots \times n_{k}}:=\mathcal{H}_{n_{1}} \otimes \mathcal{H}_{n_{2}} \otimes \ldots \otimes \mathcal{H}_{n_{k}}
$$

\subsection{Quantum measurements}

A register $X$ is a pair $(\mathcal{X}, \rho)$, where $\mathcal{X}$ is a complex Euclidean space, and $\rho \in D(\mathcal{X})$ is a density. A quantum measurement on a register $X=(\mathcal{X}, \rho)$ is a map

$$
\mu: \Gamma \rightarrow \operatorname{Pos}(\mathcal{X})
$$

where $\Gamma$ is a finite, nonempty set of measurement outcomes. To be considered a valid measurement, such a function must satisfy the constraint

$$
\sum_{a} \mu(a)=\mathbb{1}_{\mathcal{X}}
$$


It is common that one identifies the measurement $\mu$ with the collection of operators $\left\{P_{a}: a \in \Gamma\right\}$, where $P_{a}=\mu(a)$ for each $a \in \Gamma$. Each operator $P_{a}$ is called the measurement operator associated with the outcome $a \in \Gamma$.

Suppose a register $X$ is in state $\rho \in D(\mathcal{X})$. If we apply the measurement $\mu$ : $\Gamma \rightarrow \operatorname{Pos}(\mathcal{X})$, then an element $a \in \Gamma$ is randomly selected as the outcome of the measurement with the probability $p(a)$, given by

$$
p(a)=\langle\mu(a), \rho\rangle .
$$

It follows from the constraint $\sum_{a} \mu_{a}=\mathbb{1}_{\mathcal{X}}$, that the sum of these probabilities equals to 1:

$$
\sum_{a} p(a)=\sum_{a}\langle\mu(a), \rho\rangle=\left\langle\mathbb{1}_{\mathcal{X}}, \rho\right\rangle=\operatorname{Tr}(\rho)=1 .
$$

A measurement $\mu: \Gamma \rightarrow \operatorname{Pos}(\mathcal{X})$ is called a projective measurements if $\mu(a)$ is a projection for each $a \in \Gamma$. Note that the only way this can happen in the presence of the constraint $\sum_{a \in \Gamma} \mu(a)=\mathbb{1}_{\mathcal{X}}$ is for $\left\{P_{a}: a \in \Gamma\right\}$ to be projections onto mutually orthogonal subspaces of $\mathcal{X}$. When $\left\{x_{a}: a \in \Sigma\right\}$ is an orthonormal basis of $\mathcal{X}$, the projective measurement

$$
\mu: \Sigma \rightarrow \operatorname{Pos}(\mathcal{X}): a \mapsto x_{a} x_{a}^{*}
$$

is referred to as the measurement with respect to the basis $\left\{x_{a}: a \in \Sigma\right\}$.

Let $X_{1}, \ldots, X_{n}$ be distinct registers, and let $Y=\left(X_{1}, \ldots, X_{n}\right)$ be the compound register formed by viewing these $n$ registers as a single one.

A state $\rho$ of $Y$ is said to be a product state if it is the tensor product of the states $\rho_{i} \in D\left(\mathcal{X}_{i}\right)$ for $i=1,2, \ldots, n$ :

$$
\rho=\rho_{1} \otimes \ldots \otimes \rho_{n} \in D\left(\mathcal{X}_{1} \otimes \ldots \otimes \mathcal{X}_{n}\right)
$$

It represents the situation that $X_{1}, \ldots, X_{n}$ are independent, or that their states are 
independent, at a particular moment.

A product measurement is a measurement of the form

$$
\mu: \Gamma_{1} \times \ldots \times \Gamma_{n} \rightarrow \operatorname{Pos}\left(\mathcal{X}_{1} \otimes \ldots \otimes \mathcal{X}_{n}\right)
$$

defined on a register of the form $Y=\left(X_{1}, \ldots, X_{n}\right)$ for which there exist measurements

$$
\begin{gathered}
\mu_{1}: \Gamma_{1} \rightarrow \operatorname{Pos}\left(\mathcal{X}_{1}\right), \\
\vdots \\
\mu_{n}: \Gamma_{n} \rightarrow \operatorname{Pos}\left(\mathcal{X}_{n}\right),
\end{gathered}
$$

such that

$$
\mu\left(a_{1}, \ldots, a_{n}\right)=\mu_{1}\left(a_{1}\right) \otimes \ldots \otimes \mu_{n}\left(a_{n}\right)
$$

for all $\left(a_{1}, \ldots, a_{n}\right) \in \Gamma_{1} \times \ldots \times \Gamma_{n}$. A product measurement describes the situation in which the measurements $\mu_{1}, \ldots, \mu_{n}$ are independently applied to registers $\mathcal{X}_{1}, \ldots, \mathcal{X}_{n}$, and the $n$-tuple of measurement outcomes is interpreted as a single measurement outcome of the compound measurement $\mu$.

Proposition 3.2.1. [37, Proposition 3.1] Let $\mathcal{X}$ be a complex Euclidean space, and let

$$
\mu: \Gamma \rightarrow \operatorname{Pos}(\mathcal{X}) \quad: \quad a \mapsto P_{a}
$$

be a measurement on $\mathcal{X}$ with the property that the collection $\left\{P_{a}: a \in \Gamma\right\}$ spans all of $L(\mathcal{X})$. The mapping $\varphi: L(\mathcal{X}) \rightarrow \mathbb{C}^{\Gamma}$, defined by

$$
\varphi(T)(a)=\left\langle P_{a}, T\right\rangle \quad(T \in L(\mathcal{X}), a \in \Gamma)
$$

is one-to-one on $L(\mathcal{X})$. 
Given a collection of registers $\left(X_{1}, \ldots, X_{n}\right)$ in some state $\rho \in D\left(\mathcal{X}_{1} \otimes \ldots \otimes \mathcal{X}_{n}\right)$, in some cases we are only interested to extract information from only a part of such a composite system. This can be done by performing a partial measurement. For the sake of simplicity, let us define the partial measurements in the case of a pair of registers $(X, Y)$. Assume the pair has the state $\rho \in D(\mathcal{X} \otimes \mathcal{Y})$, and a measurement $\mu: \Gamma \rightarrow \operatorname{Pos}(\mathcal{X})$ is performed on $\mathcal{X}$. Conditioned on the outcome $a \in \Gamma$ resulting from this measurement, the state of $\mathcal{Y}$ will become:

$$
\frac{\operatorname{Tr}_{\mathcal{X}}\left[\left(\mu(a) \otimes 1_{\mathcal{Y}}\right) \rho\right]}{\left\langle\mu(a) \otimes 1_{\mathcal{Y}}, \rho\right\rangle} .
$$

\subsection{Reduction, extension, and purification}

Suppose that a pair of registers $(X, Y)$ has the state $\rho \in D(\mathcal{X} \otimes \mathcal{Y})$. The states of $X$ and $Y$ individually are then given by

$$
\rho^{X}=\operatorname{tr}_{\mathcal{Y}}(\rho) \quad \text { and } \quad \rho^{Y}=\operatorname{Tr}_{\mathcal{X}}(\rho)
$$

The states $\rho^{X}$ and $\rho^{Y}$ are sometimes called the reduced states of $X$ and $Y$, or the reductions of $\rho$ to $X$ and $Y$.

Suppose $(X, Y)$ is a pair of registers and it has the state $\rho \in D(\mathcal{X} \otimes \mathcal{Y})$. We say the state $\rho$ is consistent with $\sigma$ on $X$ if $\sigma=\operatorname{tr}_{\mathcal{Y}}(\rho)$. Unless $Y$ is a trivial register with just a single classical state, there are always multiple choices for $\rho$ that are consistent with $\sigma$. Any such state $\rho$ is said to be an extension of $\sigma$. For instance, $\rho=\sigma \otimes \xi$ for any density operator $\xi \in D(\mathcal{Y})$ is always an extension of $\sigma$, because

$$
\operatorname{tr}_{Y}(\sigma \otimes \xi)=\sigma \otimes \operatorname{Tr}(\mathrm{x})=\sigma
$$

If $\sigma$ is pure, this is the only possible form for an extension.

Pure states are of particular interest in quantum information theory; and a legitimate question is if given a quantum system $\mathcal{A}$ is in a mixed state, can we identify 
another system $\mathcal{B}$ such that the bipartite system $\mathcal{C}=\mathcal{A B}$ is in a pure state. The system $\mathcal{B}$ is called a reference system, is only a mathematical construction without a physical support. The process of finding a reference system given a quantum system, $\mathcal{A}$, in a mixed state, $\rho_{\mathcal{A}}$, is called purification.

More formally, let $\mathcal{H}_{A}$ and $\mathcal{H}_{B}$ be Hilbert spaces and $\rho \in \operatorname{Pos}\left(\mathcal{H}_{A}\right)$ be any positive semi-definite operator. Then a purification of $\rho$ in $\mathcal{H}_{A} \otimes \mathcal{H}_{B}$ is any vector $|\varphi\rangle$, such that

$$
\rho=\operatorname{tr}_{\mathcal{H}_{B}}(|\varphi\rangle\langle\varphi|)
$$

Theorems 3.3.1 and 3.3.2 represent the conditions for existence and also unitary equivalence of purification, respectively.

Theorem 3.3.1. [37, theorem 4.1] Let $\mathcal{H}_{A}$ and $\mathcal{H}_{B}$ be Hilbert spaces and $\rho \in \operatorname{Pos}\left(\mathcal{H}_{A}\right)$ be any positive semi-definite operator in $\mathcal{H}_{A}$. Then a purification of $\rho$ exists in $\mathcal{H}_{A} \otimes \mathcal{H}_{B}$ if and only if $\operatorname{dim} \mathcal{H}_{B} \geq \operatorname{rank}(\rho)$. Moreover, when $\operatorname{dim} \mathcal{H}_{B} \geq \operatorname{dim} \mathcal{H}_{A}$, a purification of $\rho$ always exists.

Theorem 3.3.2. [37, Theorem 4.3] Let $\mathcal{X}$ and $\mathcal{Y}$ be complex Euclidean spaces, and suppose that vectors $u, v \in \mathcal{X} \otimes \mathcal{Y}$ satisfy

$$
\operatorname{Tr}_{\mathcal{Y}}\left(u u^{*}\right)=\operatorname{Tr}_{\mathcal{Y}}\left(v v^{*}\right)
$$

There exists a unitary operator $\mathcal{U} \in U(\mathcal{Y})$ such that $v=\left(\mathbb{1}_{\mathcal{X}} \otimes \mathcal{U}\right) u$.

Quantum mechanical systems have a unique property: a bipartite system can be in a pure state for which it is not possible to assign a definite state to each of its component subsystems. This strong correlation of quantum states is called entanglement; entangled states are pure states of bipartite quantum systems. 
Example 3.3.1. Bell States. The state of a quantum system consisting of two qubits is a vector in $\mathcal{H}_{2^{2}}=\mathcal{H}_{2} \otimes \mathcal{H}_{2}$ with the orthonormal basis $\{|00\rangle,|01\rangle,|10\rangle,|11\rangle\}$ expressed as

$$
|\varphi\rangle=\alpha_{00}|00\rangle+\alpha_{01}|01\rangle+\alpha_{10}|10\rangle+\alpha_{11}|11\rangle
$$

with $\left|\alpha_{00}\right|^{2}+\left|\alpha_{01}\right|^{2}+\left|\alpha_{10}\right|^{2}+\left|\alpha_{11}\right|^{2}=1$.

Sometimes the state of a two-qubit system can be factored as the tensor product of the individual states of two qubits. For example, when $\alpha_{00}=\alpha_{10}=1 / 2$ and $\alpha_{01}=\alpha_{11}=-i / 2$ the state is the tensor product of the states of the two qubits, $\left|\varphi_{1}\right\rangle$ and $\left|\varphi_{2}\right\rangle$ :

$$
\begin{gathered}
|\varphi\rangle=\frac{1}{2}[|00\rangle-i|01\rangle+|10\rangle-i|11\rangle]=\frac{1}{2}[|0\rangle \otimes(|0\rangle-i|1\rangle)+|1\rangle \otimes(|0\rangle-i|1\rangle)] \\
=\frac{1}{2}(|0\rangle+|1\rangle) \otimes(|0\rangle-i|1\rangle)=\left|\varphi_{1}\right\rangle \otimes\left|\varphi_{2}\right\rangle .
\end{gathered}
$$

This factorization is not always feasible. For example, consider a special state of a two-qubit system when

$$
\alpha_{00}=\alpha_{11}=\frac{1}{2} \quad \text { and } \quad \alpha_{01}=\alpha_{10}=0
$$

The resulting state $\left|\beta_{00}\right\rangle$ is called a Bell state and the pair of qubits is called an EPR pair

$$
\beta_{00}=\frac{|00\rangle+|11\rangle}{\sqrt{2}}
$$

There are three other Bell states

$$
\beta_{01}=\frac{|01\rangle+|10\rangle}{\sqrt{2}}, \quad \beta_{10}=\frac{|00\rangle-|11\rangle}{\sqrt{2}}, \quad \text { and } \quad \beta_{11}=\frac{|01\rangle-|10\rangle}{\sqrt{2}} \text {. }
$$

These states form an orthonormal basis and can be distinguished from one another. Bell states are entangled states; the four states are called maximally entangled states 
and $\left|\beta_{11}\right\rangle$ is an anticorrelated state.

\subsection{Quantum channels}

Quantum channels represent the physical medium that enable the transfer of a quantum system from a sender to a receiver. Since quantum systems are described mathematically by density operators, quantum channels should be mathematical objects that transform densities.

We denote by $\mathcal{T}(\mathcal{X}, \mathcal{Y})$ the space of all linear mappings of the form $\Phi: L(\mathcal{X}) \rightarrow$ $L(\mathcal{Y})$. A mapping $\Phi \in T(\mathcal{X}, \mathcal{Y})$ is said to be positive if $\Phi(P) \in \operatorname{Pos}(\mathcal{Y})$ for all $P \in \operatorname{Pos}(\mathcal{X})$, and is said to be completely positive if and only if $\Phi \otimes \mathbb{1}_{L(\mathcal{Z})}$ is positive for every choice of a complex Euclidean space $\mathcal{Z}$.

Definition 3.4.1. A quantum channel from a register $X$ to a register $Y$ is a linear mapping of the form

$$
\Phi: L(\mathcal{X}) \rightarrow L(\mathcal{Y})
$$

that satisfies two restrictions:

(i) $\Phi$ must be trace-preserving, and

(ii) $\Phi$ must be completely positive.

A simple example of quantum channels is the identity channel $\mathbb{1}_{L(\mathcal{X})}$, which leaves each $T \in L(\mathcal{X})$ unchanged.

In the following we will introduce four representation of such mappings which are: the natural representation, the Choi-Jamiolkowski representation, the Kraus representations, and the Stinespring representations.

We borrow the following from [37]. 
We start with the natural representation. For every mapping $\Phi \in \mathcal{T}(\mathcal{X}, \mathcal{Y})$, it is clear that the mapping

$$
\operatorname{vec}(X) \rightarrow \operatorname{vec}(\Phi(X))
$$

is linear, as it can be represented as a composition of linear mappings. To be precise, there must exist a linear operator $K(\Phi) \in L(\mathcal{X} \otimes \mathcal{X}, \mathcal{Y} \otimes \mathcal{Y})$ that satisfies

$$
K(\Phi) \operatorname{vec}(X)=\operatorname{vec}(\Phi(X))
$$

for all $X \in L(\mathcal{X})$. The operator $K(\Phi)$ is called the natural representation of $\Phi$. A concrete expression for $K(\Phi)$ is as follows:

$$
K(\Phi)=\sum_{a, b \in \Sigma} \sum_{c, d \in \Gamma}\left\langle E_{c, d}, \Phi\left(E_{a, b}\right)\right\rangle E_{c, a} \otimes E_{d, b}
$$

The natural representation satisfies the following properties:

(i) The mapping $K: \mathcal{T}(\mathcal{X}, \mathcal{Y}) \rightarrow L(\mathcal{X} \otimes \mathcal{X}, \mathcal{Y} \otimes \mathcal{Y})$ is itself linear:

$$
K(\alpha \Phi+\beta \Psi)=\alpha K(\Phi)+\beta K(\Psi)
$$

for all choices of $\alpha, \beta \in C$ and $\Phi, \Psi \in \mathcal{T}(\mathcal{X}, \mathcal{Y})$.

(ii) $K$ is a linear bijection, for every operator $A \in L(\mathcal{X} \otimes \mathcal{X}, \mathcal{Y} \otimes \mathcal{Y})$ there exists a unique choice of $\Phi \in \mathcal{T}(\mathcal{X}, \mathcal{Y})$ for which $A=K(\Phi)$.

(iii) The natural representation respects the notion of adjoints, meaning that

$$
K\left(\Phi^{*}\right)=(K(\Phi))^{*}
$$

for every mapping $\Phi \in \mathcal{T}(\mathcal{X}, \mathcal{Y})$ 
The next representation is the Choi-Jamiolkowski representation which is very important in understanding completely positive mappings in particular. Specifically, let us define a mapping $J: \mathcal{T}(\mathcal{X}, \mathcal{Y}) \rightarrow L(\mathcal{Y} \otimes \mathcal{X})$ as

$$
J(\Phi)=\sum_{a, b \in S} \Phi\left(E_{a, b}\right) \otimes E_{a, b}
$$

for each $\Phi \in \mathcal{T}(\mathcal{X}, \mathcal{Y})$. The operator $J(\Phi)$ is called the Choi-Jamiolkowski representation of $\Phi$.

As for the natural representation, it is evident from the definition that $J$ is a linear bijection. Mapping $\Phi$ can be recovered from the operator $J(\Phi)$ by means of the equation

$$
\Phi(T)=\operatorname{tr}_{\mathcal{X}}\left[J(\Phi)\left(\mathbb{1}_{\mathcal{Y}} \otimes T^{t}\right)\right]
$$

Another representation for quantum channels is the Kraus representation. Let $\Phi \in$ $\mathcal{T}(\mathcal{X}, \mathcal{Y})$ be a mapping, and suppose that $\left\{A_{a}: a \in \Gamma\right\},\left\{B_{a}: a \in \Gamma\right\} \subset L(\mathcal{X}, \mathcal{Y})$ are (finite and nonempty) collections of operators for which the equation

$$
\Phi(T)=\sum_{a \in \Gamma} A_{a} T B_{a}^{*}
$$

holds for all $T \in L(\mathcal{X})$. The above expression is said to be a Kraus representation of the mapping $\Phi$. It will be established shortly that Kraus representations exist for all mappings. Unlike the natural representation and Choi-Jamiolkowski representation, Kraus representations are never unique. The term Kraus representation is sometimes reserved for the case that $A_{a}=B_{a}$ for each $a \in \Gamma$, and in this case the operators $\left\{A_{a}: a \in \Gamma\right\}$ are called Kraus operators. Such a representation exists, as we will see shortly, if and only if $\Phi$ is completely positive. Under the assumption that $\Phi$ is given 
by the mentioned equation, we have

$$
\Phi^{*}(\mathcal{S})=\sum_{a \in \Gamma} A_{a}^{*} S B_{a}
$$

for all $S \in L(\mathcal{Y})$.

Finally, for the Stinespring representations suppose that $\Phi \in \mathcal{T}(\mathcal{X}, \mathcal{Y})$ is a given mapping, $\mathcal{Z}$ is a complex Euclidean space, and $A, B \in L(\mathcal{X}, \mathcal{Y} \otimes \mathcal{Z})$ are operators such that

$$
\Phi(T)=\operatorname{tr}_{\mathcal{Z}}\left(A T B^{*}\right)
$$

for all $T \in L(\mathcal{X})$. Such a representation is called a Stinespring representation of $\Phi$. Similar to Kraus representations, Stinespring representations always exists for a given $\Phi$ and are never unique.

Similar to Kraus representations, the term Stinespring representation is often reserved for the case $A=B$. Once again, as we will see, such a representation exists if and only if $\mathrm{F}$ is completely positive. If $\Phi \in \mathcal{T}(\mathcal{X}, \mathcal{Y})$ is given by the above equation, then

$$
\Phi^{*}(S)=A^{*}\left(S \otimes \mathbb{1}_{\mathcal{Z}}\right) B
$$

The following proposition explains a simple way in which the four representations discussed above relate to one another.

Proposition 3.4.1. [37, Proposition 5.2] Let $\Phi \in \mathcal{T}(\mathcal{X}, \mathcal{Y})$ and let $\left\{A_{a}: a \in \Gamma\right\}$ and $\left\{B_{a}: a \in \Gamma\right\}$ be collections of operators in $L(\mathcal{X}, \mathcal{Y})$ for a finite, non-empty set $\Gamma$. The following statements are equivalent:

1. (Kraus representations.) We have

$$
\Phi(T)=\sum_{a \in \Gamma} A_{a} T B_{a}^{*}
$$


for all $T \in L(\mathcal{X})$.

2. (Stinespring representations.) For $\mathcal{Z}=\mathbb{C}^{\Gamma}$ and $A, B \in L(\mathcal{X}, \mathcal{Y} \otimes \mathcal{Z})$ defined as

$$
A=\sum_{a \in \Gamma} A_{a} \otimes e_{a} \text { and } B=\sum_{a \in \Gamma} B_{a} \otimes e_{a}
$$

We have $\Phi(T)=\operatorname{tr}_{\mathcal{Z}}\left(A T B^{*}\right)$ for all $T \in L(\mathcal{X})$.

3. (The natural representation.) We have

$$
K(\Phi)=\sum_{a \in \Gamma} A_{a} \otimes \overline{B_{a}} .
$$

4. (The Choi-Jamiolkowski representation.) We have

$$
J(\Phi)=\sum_{a \in \Gamma} \operatorname{vec}\left(A_{a}\right) \operatorname{vec}\left(B_{a}^{*}\right) .
$$

Theorems 3.4.1 and 3.4.2 represent the characterizations of completely positive and trace-preserving maps.

Theorem 3.4.1. [37, Theorem 5.3] For every mapping $\Phi \in \mathcal{T}(\mathcal{X}, \mathcal{Y})$, the following statements are equivalent:

1. $\Phi$ is completely positive.

2. $\Phi \otimes \mathbb{1}_{L(\mathcal{X})}$ is positive.

3. $J(\Phi) \in \operatorname{Pos}(\mathcal{Y} \otimes \mathcal{X})$.

4. There exists a finite set of operators $\left\{A_{a}: a \in \Gamma\right\} \subset L(\mathcal{X}, \mathcal{Y})$ such that

$$
\Phi(T)=\sum_{a \in \Gamma} A_{a} T A_{a}^{*}
$$


for all $T \in L(\mathcal{X})$.

5. Item 4 holds for $|\Gamma|=\operatorname{rank}(J(\Phi))$.

6. There exists a complex Euclidean space $\mathcal{Z}$ and an operator $A \in L(\mathcal{X}, \mathcal{Y} \otimes \mathcal{Z})$ such that

$$
\Phi(T)=\operatorname{tr}_{\mathcal{Z}}\left(A T A^{*}\right)
$$

for all $T \in L(\mathcal{X})$.

7. Item 6 holds for $\mathcal{Z}$ having dimension equal to the rank of $J(\Phi)$.

Theorem 3.4.2. [37, Theorem 5.4] For every mapping $\Phi \in T(\mathcal{X}, \mathcal{Y})$, the following statements are equivalent:

1. $\Phi$ is trace-preserving.

2. $\Phi^{*}$ is unital (i.e. $\left.\Phi^{*}\left(1_{\mathcal{X}}\right)=\mathbb{1}_{\mathcal{Y}}\right)$.

3. $\operatorname{tr}_{\mathcal{Y}}(J(\Phi))=\mathbb{1}_{\mathcal{X}}$.

4. There exists a Kraus representation

$$
\Phi(T)=\sum_{a \in \Gamma} A_{a} T B_{a}^{*}
$$

of $\Phi$, for which the operators $\left\{A_{a}: a \in \Gamma\right\},\left\{B_{a}: a \in \Gamma\right\} \subset L(\mathcal{X}, \mathcal{Y})$ satisfy

$$
\sum_{a \in \Gamma} A_{a}^{*} B_{a}=\mathbb{1}_{\mathcal{X}} .
$$

5. For all Kraus representations

$$
\Phi(T)=\sum_{a \in \Gamma} A_{a} T B_{a}^{*}
$$


of $\Phi$, the operators $\left\{A_{a}: a \in \Gamma\right\},\left\{B_{a}: a \in \Gamma\right\} \subset L(\mathcal{X}, \mathcal{Y})$ satisfy

$$
\sum_{a \in \Gamma} A_{a}^{*} B_{a}=\mathbb{1}_{\mathcal{X}}
$$

6. There exists a Stinespring representation

$$
\Phi(T)=\operatorname{tr}_{\mathcal{Z}}\left(A T B^{*}\right)
$$

of $\Phi$ for which the operators $A, B \in L(\mathcal{X}, \mathcal{Y} \otimes \mathcal{Z})$ satisfy $A^{*} B=\mathbb{1}_{\mathcal{X}}$.

7. For all Stinespring representations

$$
\Phi(T)=\operatorname{tr}_{\mathcal{Z}}\left(A T B^{*}\right)
$$

of $\Phi$, the operators $A, B \in L(\mathcal{X}, \mathcal{Y} \otimes \mathcal{Z})$ satisfy $A^{*} B=\mathbb{1}_{\mathcal{X}}$.

Hence we obtain the following characterization of quantum channels.

Corollary 3.4.1. [37, Corollary 5.5] Let $\Phi \in \mathcal{T}(\mathcal{X}, \mathcal{Y})$. The following statements are equivalent:

1. $\Phi$ is a quantum channel, i.e. completely positive and trace-preserving.

2. $J(\Phi) \in \operatorname{Pos}(\mathcal{Y} \otimes \mathcal{X})$ and $\operatorname{Tr}_{\mathcal{Y}}(J(\Phi))=\mathbb{1}_{\mathcal{X}}$.

3. There exists a finite set of operators $\left\{A_{a}: a \in \Gamma\right\} \subset L(\mathcal{X}, \mathcal{Y})$ such that

$$
\Phi(T)=\sum_{a \in \Gamma} A_{a} T A_{a}^{*}
$$


for all $T \in L(\mathcal{X})$, and such that

$$
\sum_{a \in \Gamma} A_{a}^{*} A_{a}=\mathbb{1}_{\mathcal{X}}
$$

4. Item 3 holds for $\Gamma$ satisfying $|\Gamma|=\operatorname{rank}(J(\Phi))$.

5. There exists a complex Euclidean space $\mathcal{Z}$ and a linear isometry $A \in U(\mathcal{X}, \mathcal{Y} \otimes \mathcal{Z})$, such that

$$
\Phi(T)=\operatorname{tr}_{\mathcal{Z}}\left(A T A^{*}\right)
$$

for all $T \in L(\mathcal{X})$.

6. Item 5 holds for a complex Euclidean space $\mathcal{Z}$ with $\operatorname{dim}(\mathcal{Z})=\operatorname{rank}(J(\Phi))$.

A quantum channel is noiseless when the quantum system is undisturbed during the transfer and it is noisy when the quantum system interacts with some other system, e.g., the environment, during the transfer. Noise is an undesirable transformations on a quantum system during the transfer from the sender to the receiver. There are several types of quantum operations carried out by noisy quantum channels like state depolarization, amplitude.

Example 3.4.1. Consider a quantum system $A \in \mathcal{H}_{n}$ with density matrix, $\rho^{\mathcal{A}}$. A depolarizing channel characterized by the quantum operation $N$ replaces $\mathcal{A}$ with the completely mixed state $\frac{1}{n} I$, with probability $p$ and leaves the system's state unchanged with probability $(1-p)$ :

$$
N\left(\rho^{\mathcal{A}}\right)=\frac{p}{n} I+(1-p) \rho^{\mathcal{A}} .
$$




\section{Chapter 4}

\section{Classical error correction}

A communication channel is an abstract apparatus used for transmitting information from one place or time to another place or time, called the transmitter, and the receiver respectively.

In order to transmit information through communication channels we need to code our information into a language understandable for the channel.

A channel alphabet is a finite set $\mathcal{A}$ of symbols which can be transmitted through a channel. If $|\mathcal{A}|=2$, then $\mathcal{A}=\{0,1\}$ is called the binary alphabet.

A finite sequence of elements from a channel alphabet is called a codeword.

\subsection{Error detection and error correction}

Real life communication channels are usually noisy. Error detecting is the process of determining if a message that is being transmitted through a noisy channel is indeed in error. And error correcting is the process of returning damaged messages to their original, error-free contents. The main idea behind error correction methods is based on increasing the redundancy of the message. 
Redundancy is the amount of extra space used to transmit a message, i.e. the difference between the number of the bits of actual information and the number of the bits used to transmit it.

A good code should add a minimum amount of redundancy to actual information.

Example 4.1.1. To illustrate the idea of redundancy, we give a very simple example. Suppose we need to send a single bit of information through a noisy channel. Further suppose that this single bit is subject to at most one possible error that may cause it changing from 0 to 1 , or vice versa (the bit flip error). The idea of the coding method is that instead of sending a single bit we send 3 copies of the bit into the channel, i.e. a string of length 3 .

$$
\begin{aligned}
& 0 \rightarrow(000) ; \\
& 1 \rightarrow(111) .
\end{aligned}
$$

In order to decode the codeword we use the majority voting method: if we receive the 3-tuples (011), for example, we simply decode it as 1.

More generally, if at most $k$ number of bit-flips can be caused by the channel, we make a string of $n=2 k+1$ copies of the bit containing the information. If the number of 0's in the outcome is greater than $k$ we decode it as 0 , otherwise we decode it as 1 .

To provide a real-life coding scheme, we give the example of the International Standard Book Number (ISBN), which allows error detection.

Example 4.1.2. The International Standard Book Number (ISBN) coding scheme is a unique numeric commercial book identifier based upon 10 digits:

$$
\begin{array}{llllllllll}
d_{1} & d_{2} & d_{3} & d_{4} & d_{5} & d_{6} & d_{7} & d_{8} & d_{9} & d_{10},
\end{array}
$$


where $d_{10}$, which is called the check digit, is a form of redundancy used for error detection, and is computed from the first nine digits in the following way:

$$
10 d_{1}+9 d_{2}+8 d_{3}+7 d_{4}+6 d_{5}+5 d_{6}+4 d_{7}+3 d_{8}+2 d_{9}+d_{10} \equiv 0(\bmod 11) .
$$

This code can detect any single-digit error or one replacement of adjacent digits. These are indeed the two most common errors that occur in handling an ISBN (e.g. typing or writing it). If either of these types of error has occurred, the result will never be a valid ISBN. In fact the sum of the digits multiplied by their weights will never be a multiple of 11 .

A communication channel model consists of a source encoder, a compressor, a channel encoder, a channel, a channel decoder, an expander, and a source decoder. Since our focus in this thesis is on error detection and error correction, we ignore the parts corresponding to data compression, i.e. the compressor and the expander.

The source encoder transforms the information into $k$-tuples of the code alphabet. The channel encoder then transform those $k$-length codes to longer codes of $k+r$ digits, i.e. adding redundancy. The channel transmits the $(k+r)$-symbols from one place (or/and time) to another place (or/and time). The channel decoder maps the outcome $(k+r)$-tuples back to $k$-tuples. And finally, the source decoder restores the message.

A block code is a one-to-one mapping $B: \mathcal{A}^{k} \rightarrow \mathcal{A}^{n}$, where $\mathcal{A}$ is the source alphabet, and $k$ and $n$ are integers. The ratio $k / n$ is called the rate of the block code $B$.

Definition 4.1.1. The Hamming distance between two vector $x=\left(x_{1}, \ldots, x_{n}\right)$ and 
$y=\left(y_{1}, \ldots, y_{n}\right)$ is defined by

$$
d_{\text {Hamming }}(x, y):=\left|\left\{i: 1 \leq i \leq n: x_{i}=y_{i}\right\}\right| .
$$

For instance, the Hamming distance between two 5-tuples $v=(10001)$ and $u=$ (01100) is 4. The Hamming distance is used to measure the similarity (or equivalently the difference) of two given codewords.

The Hamming sphere around $v$ of radius $r$ is:

$$
S=\{u \in H ; d(v, u) \leq r\}
$$

Example 4.1.3. The Hamming sphere around the 4-tuples (1111) and (0011) of radius 1 , denoted by $S_{1}$ and $S_{2}$ respectively, are:

$$
\begin{aligned}
& S_{1}=\{(1111),(0111),(1011),(1101),(1110)\}, \\
& S_{2}=\{(0011),(0111),(1011),(0001),(0010)\} .
\end{aligned}
$$

The minimum distance of a block code $B$ is the minimum number of positions in which two distinct codewords differ, i.e.

$$
d_{\min }(B):=\min \left\{d_{\text {Hamming }}(x, y): x, y \in B \text { and } x \neq y\right\} .
$$

\subsubsection{Decoding}

The error detection and error correction processes, which are done by the decoder, are as follows: first, the decoder checks whether the received $n$-tuple is among the valid codewords (i.e. the ones that used by the channel encoder to code a piece of 
information). If that is the case, no error has occurred, and the output is decoded as is. But if the decoder could not find any similar codeword, it concludes that an error has occurred. The next step for decoder is then correcting the error. For this the decoder finds the codeword which has the least Hamming distance to the received $n$-tuple, and use that codeword to decode the output.

A very serious problem with this system of error detection is that, in case an error has occurred and changed a valid codeword into another valid one it is impossible for the system to detect the error.

The following theorem provides a manner to assess the error-correcting capability of a given block code.

Theorem 4.1.1. Let $B$ be a block code with minimum Hamming distance $d$. Then one can either detect any error that acts on no more than d positions or correct any error that acts on no more than $\lfloor(d-1) / 2\rfloor$ positions.

Proof. From the definition of the minimum distance of the code $B$ it follows that at least $d$ positions have to be changed in oder to transform one codeword into another. Hence any error acting on less than $d-1$ positions can be detected. If strictly less than $d / 2$ positions are changed, there will be a unique codeword which is closest in the Hamming distance. Hence up to $\lfloor(d-1) / 2\rfloor$ errors can be corrected.

The idea behind classical error correction is to first locate $2^{k}$ Hamming spheres with radius $t$ in a $2^{n}$-dimensional Hilbert Space $\mathcal{H}$ such that each possible codeword is contained in one, and only one sphere. We then choose the centres of these spheres as codewords.

It is obviously seen that such a coding scheme can correct up to $t$ errors in the following way. After receiving the outcome $n$-tuple, we decode it as the centre of the sphere containing it. 
Note that this coding scheme can correct the $n$-tuples which do not have more than $t$ (the radius of the Hamming sphere) errors. 


\section{Chapter 5}

\section{Quantum error correction}

In this chapter we present the main content of this thesis. We introduce the concept of quantum error, and quantum error correction. We give concrete examples of quantum error correcting schemes. The main methods of quantum error correction, the standard model, the decoherence subspace, and the noiseless subsystem methods are introduced, and their mathematical formalisms are presented. We give necessary and sufficient conditions under which each method is applicable to a given quantum system with a specific noise operator.

We conclude the chapter, and hence the thesis in the last section by presenting the operator quantum error correction method, a generalized approach developed by Kribs, Laflamme, and Poulin [21], that in fact unifies other previously existing schemes of error correcting.

\subsection{Quantum errors}

The only type of error that occurs in classical systems is the bit-flip, which changes the state of the bit from 0 to 1 and vice versa. But in a quantum system, in addition 
to the possible bit-flip, an error may also be caused by the phase-flip.

Consider the qubit $|\phi\rangle$ in the state

$$
|\phi\rangle=\alpha|0\rangle+\beta|1\rangle
$$

The error caused by the bit-flip changes the state of $|\phi\rangle$ to

$$
\alpha|0\rangle+\beta|1\rangle \leadsto \beta|1\rangle+\alpha|0\rangle
$$

This error is in fact results from the application of the Pauli operator $\sigma_{x}$ on $|\phi\rangle$ :

$$
\sigma_{x}|\phi\rangle=\left(\begin{array}{ll}
0 & 1 \\
1 & 0
\end{array}\right)\left(\begin{array}{l}
\alpha \\
\beta
\end{array}\right)=\left(\begin{array}{l}
\beta \\
\alpha
\end{array}\right) .
$$

The error caused by the phase-flip changes the state of $|\phi\rangle$ to

$$
\alpha|0\rangle+\beta|1\rangle \leadsto \alpha|0\rangle-\beta|1\rangle
$$

And the Pauli operator causing this error is $\sigma_{z}$ :

$$
\sigma_{z}|\phi\rangle=\left(\begin{array}{cc}
1 & 0 \\
0 & -1
\end{array}\right)\left(\begin{array}{l}
\alpha \\
\beta
\end{array}\right)=\left(\begin{array}{c}
\alpha \\
-\beta
\end{array}\right)
$$

Another quantum error which is indeed the combination of these two types of error is the bit- \& phase-flip, that changes the state of the system in the following way:

$$
\alpha|0\rangle+\beta|1\rangle \leadsto \beta|0\rangle-\alpha|1\rangle
$$


and obviously, the Pauli operator of this error is $-i \sigma_{y}=\sigma_{x} \sigma_{z}$ :

$$
-i \sigma_{y}|\phi\rangle=\left(\begin{array}{cc}
0 & -1 \\
1 & 0
\end{array}\right)\left(\begin{array}{l}
\alpha \\
\beta
\end{array}\right)=\left(\begin{array}{c}
\beta \\
-\alpha
\end{array}\right) \text {. }
$$

Errors in a quantum system are in fact linear combination of the above basic quantum errors.

\subsection{Error correction: classical vs. quantum}

The concept of quantum error correction is fundamentally built on the ideas of the classical error correction. In fact, quantum error correcting methods are mainly based on the classical error correcting techniques.

This of course does not mean we can apply classical error correcting methods to the quantum case, in the exact same way. Need for different techniques to correct quantum errors is inevitable because of the fundamental structural differences between quantum systems and classical ones. Here are some of these differences:

No-Cloning Theorem: Classical error correcting methods use redundancy to protect a transmitting bit from error. But doing the same is not possible in a Quantum system according to the "No-Cloning theorem" [40], that implies that there cannot be such a machine that perform $|\phi\rangle \rightarrow|\phi\rangle \otimes|\phi\rangle$.

Measurement: According the axioms of quantum mechanics, measurement disturbs the state of a quantum system. Therefore, we cannot measure the quantum system to detect the errors. So we need to find methods that provide us with a way of error detection without destroying the state of the quantum system.

Entanglement: Another special property of quantum system is entanglement, which does not have any classical equivalence. Most errors occur when the state of a quan- 
tum system gets entangled with the environment. This phenomenon causes new challenges in error correction for quantum information, but also, as we will see later, provides new possibilities of using the power of entanglement to design quantum correcting codes.

Due to implications of the axioms of quantum mechanics, and the resulting challenges that they impose on the concept of error correction, it was believed impossible to correct Quantum errors, before Shor presented in 1995 a code that could correct bit-flip and phase-flip errors on a single qubit [32]. Since any method of error correction requires a way of error correction, that can only be done through a measurement, the destructive effect of measurements in quantum systems was the main source of doubts about the possibility of quantum error correction. But in his genius error correcting scheme, Shor shows that it is possible to detect an error by only partial knowledge of the state of the quantum computer, hence by performing only non-destructive partial measurements on the system. It is worth mentioning that the main tool in his method is the entanglement.

More efficient error correcting codes were constructed later, using Shor's original idea and method. Stean presented a code which does the same by using only seven qubits instead of nine [35]. Laflamme found the smallest possible code, which protects a single logical qubit against possible errors, by a five qubits code.

Next section contains more details and some concrete examples.

\subsection{Active methods of quantum error correction}

We demonstrate Shor's main idea in his original error correcting code in the following simple example. In fact, to see how this method of error correction works in details, let us consider the simplest possible case: correcting a bit-flip error on a qubit via a 
3-qubit code.

Example 5.3.1. In this setup, a single qubit $|\varphi\rangle$ in the state $|\varphi\rangle=\alpha|0\rangle+\beta|1\rangle$ is to be transmitted through a communication channel, subject to one possible bit-flip error. The main idea of this coding scheme is to first encode 1-qubits to 3-qubits to create more resilient information, then add 2 more ancilla qubits to the codeword to help detecting the error. Using appropriate CNOT gates, ancilla qubits are entangled with the state of the system in a away that they change according to the type of error applied to the original state of the system. Then partial measurement on the system detects from ancilla vectors what the error is, and then a reverse operation will correct the original state.

More precisely, we encode each single logical qubit into 3-physical qubit:

$$
\begin{gathered}
|0\rangle \rightarrow|0\rangle_{L}=|000\rangle, \\
|1\rangle \rightarrow|1\rangle_{L}=|111\rangle .
\end{gathered}
$$

And therefore

$$
\alpha|0\rangle+\beta|1\rangle \rightarrow \alpha|000\rangle+\beta|111\rangle=\alpha|0\rangle_{L}+\beta|1\rangle_{L}
$$

In the following table the state of the ancilla qubits after the effect of bit-flip in data block is given.

\begin{tabular}{|l|l|}
\hline No Error & $\alpha|000\rangle|00\rangle+\beta|111\rangle|00\rangle$ \\
\hline Qubit 1 & $\alpha|100\rangle|11\rangle+\beta|011\rangle|11\rangle$ \\
\hline Qubit 2 & $\alpha|010\rangle|10\rangle+\beta|101\rangle|10\rangle$ \\
\hline Qubit 3 & $\alpha|001\rangle|01\rangle+\beta|110\rangle|01\rangle$ \\
\hline
\end{tabular}

For each possible situation, either no error or a single bit-flip error, the ancilla 
qubits are flipped to a unique state accordingly. In this model, we assume that the interaction with environment is independent with each qubit. So the Unitary operator which we use for recovering the data is the tensor product of operators that we should apply on each qubit separately:

\begin{tabular}{|l|l|}
\hline Error & Unitary Operator \\
\hline No Error & $C_{0}=I \otimes I \otimes I$ \\
\hline Bit-flip error in first qubit & $C_{1}=X \otimes I \otimes I$ \\
\hline Bit-flip error in second qubit & $C_{2}=I \otimes X \otimes I$ \\
\hline Bit-flip error in third qubit & $C_{3}=I \otimes I \otimes X$ \\
\hline
\end{tabular}

The following is the quantum circuit for this correcting scheme. (In a quantum circuit, horizontal lines represent qubits, vertical lines represent CNOT gates, with dark circles determining the control qubit, and rectangles represent quantum gates, through which qubits undergo changes.)

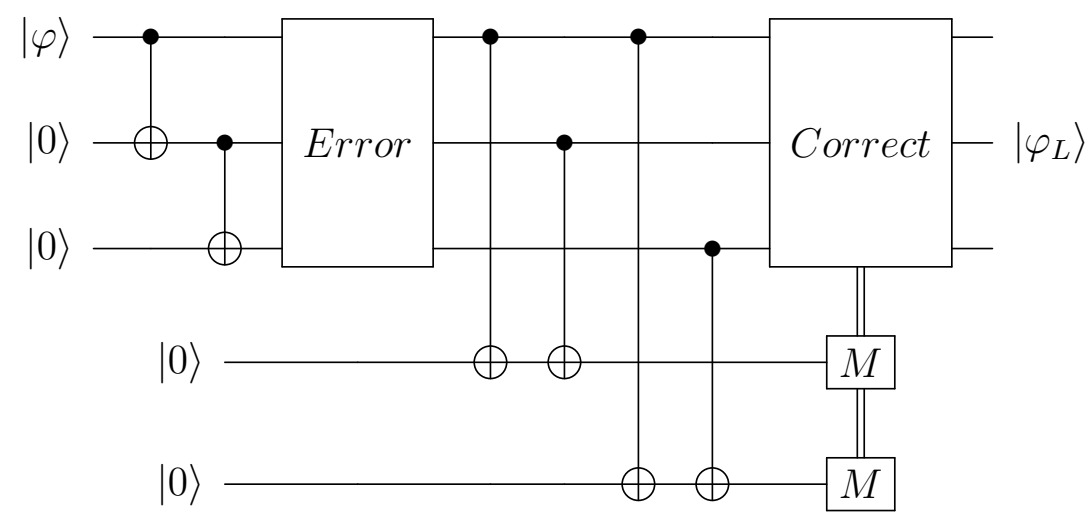


Example 5.3.2. Next consider the case of a possible phase-flip error. We can transform this setup to the previous situation by performing an appropriate change of basis. More precisely, applying the Hadamard gate results to the change of basis

$$
\begin{aligned}
& |0\rangle \leadsto \frac{1}{\sqrt{2}}(|0\rangle+|1\rangle), \\
& |1\rangle \leadsto \frac{1}{\sqrt{2}}(|0\rangle-|1\rangle) .
\end{aligned}
$$

Obviously a phase-flip in the first basis corresponds to a bit-flip in the second.

Example 5.3.3. [The Shor Code] Shor's nine-qubits quantum error correcting code can correct a single bit-flip error, a single phase-flip error, or a single bit- \& phase-flip error.

First, a qubit is encoded for phase-flip error correction, then each of the resulting three qubits is encoded for bit-flip error correction. The two basis vectors in $\mathcal{H}_{2}$, $\{|0\rangle,|1\rangle\}$ are transformed in $\left\{|0\rangle_{L},|1\rangle_{L}\right\}$ in $\mathcal{H}_{2^{9}}$ as follows

$$
\begin{gathered}
|0\rangle \leadsto|+++\rangle=\frac{|0\rangle+|1\rangle}{\sqrt{2}} \otimes \frac{|0\rangle+|1\rangle}{\sqrt{2}} \otimes \frac{|0\rangle+|1\rangle}{\sqrt{2}} \quad \text { Phase-flip } \\
\sim|0\rangle_{L}=\frac{|000\rangle+|111\rangle}{\sqrt{2}} \otimes \frac{|000\rangle+|111\rangle}{\sqrt{2}} \otimes \frac{|000\rangle+|111\rangle}{\sqrt{2}} \quad \text { Bit-flip; } \\
\qquad 1\rangle \sim|---\rangle=\frac{|0\rangle-|1\rangle}{\sqrt{2}} \otimes \frac{|0\rangle-|1\rangle}{\sqrt{2}} \otimes \frac{|0\rangle-|1\rangle}{\sqrt{2}} \quad \text { Phase-flip } \\
\leadsto|1\rangle_{L}=\frac{|000\rangle-|111\rangle}{\sqrt{2}} \otimes \frac{|000\rangle-|111\rangle}{\sqrt{2}} \otimes \frac{|000\rangle-|111\rangle}{\sqrt{2}} \quad \text { Bit-flip. }
\end{gathered}
$$


The quantum circuit of this scheme is

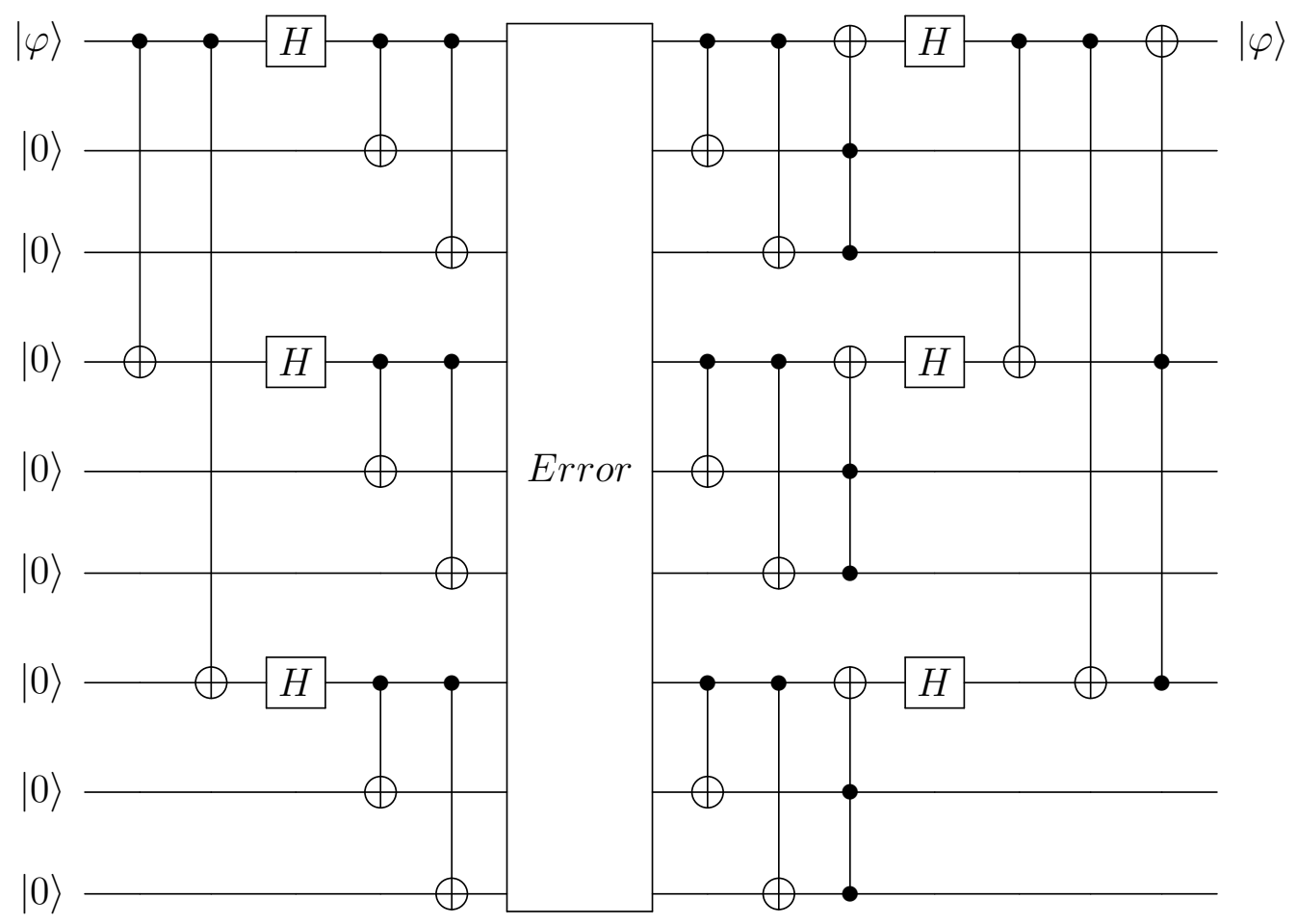

\subsubsection{Mathematical formalism}

Suppose the information is stored in the initial state $\rho_{i}$ of the quantum system. Interaction with environment may result in errors, and hence change the state $\rho_{i}$. Let $\mathcal{E}$ denote this error operator resulting from interaction with the environment. Then the state of the system after the interaction with environment will be

$$
\rho=\mathcal{E}\left(\rho_{i}\right)
$$


The error operator $\mathcal{E}$ is in fact itself a quantum channel, hence represented by its Kraus operators $\left\{A_{a}\right\}$, called the interaction operators:

$$
\rho=\mathcal{E}\left(\rho_{i}\right)=\sum_{a} A_{a} \rho_{i} A_{a}^{*} .
$$

The interaction operators can be obtained by knowing the initial state of the environment, $|e\rangle$, the orthonormal basis of the environment, $\left|e_{a}\right\rangle$, and also the unitary $U$ that implements the time evolution of the system:

$$
A_{a}=\left\langle e_{a}|U| e\right\rangle .
$$

In the setup we consider in this section, we always assume that qubits have independent environment, i.e. error affect each qubit independently.

For $k, n \in \mathbb{N}$, we define a quantum code $\mathcal{C}$ with the rate $k / n$ to be a unitary mapping from $\mathcal{H}_{k}$ onto the $k$-dimensional subspace $\mathcal{C} \leq \mathcal{H}_{2^{n}}$. That is, a quantum code with rate $k / n$ is determined by a $k$-dimensional subspace of a $2^{n}$-dimensional Hilbert space. By a recovery operator for a quantum code $\mathcal{C}$ as above, we mean an operator $\mathcal{R}: \mathcal{B}\left(\mathcal{H}_{2^{n}}\right) \rightarrow \mathcal{B}(\mathcal{C})$. (Here, and in the rest of this section we denote by $\mathcal{B}(\mathcal{H})$ the space of all linear maps on a finite-dimensional Hilbert space $\mathcal{H}$.)

Theorem 5.3.1. [17, Theorem III.3-III.5] Let $\mathcal{E}$ be a quantum channel on $\mathcal{H}, \mathcal{C} \leq \mathcal{H}$ be a quantum code, and let $\mathcal{R}$ be recovery operator for $\mathcal{C}$. Suppose $\left\{A_{a}\right\}$ and $\left\{R_{r}\right\}$ are Kraus operators of $\mathcal{E}$ and $\mathcal{R}$, respectively. Then the followings are equivalent:

1. There exist $\lambda_{r a} \in \mathbb{C}$ such that

$$
P_{\mathcal{C}} R_{r} A_{a} P_{\mathcal{C}}=\lambda_{r, a} P_{\mathcal{C}}
$$

where $P_{\mathcal{C}}$ is the orthogonal projection onto $\mathcal{C}$. 
2. When restricted to $\mathcal{C}$, a multiple of $\mathcal{R}$ is the left inverse of $\mathcal{E}$, i.e. there is $\lambda>0$ such that

$$
\mathcal{R} \mathcal{E}\left(P_{\mathcal{C}} T P_{\mathcal{C}}\right)=\lambda P_{\mathcal{C}} T P_{\mathcal{C}}
$$

for all $T \in \mathcal{B}(\mathcal{H})$.

3. If $\left\{\left|e_{i}\right\rangle\right\}$ is an orthonormal basis for $\mathcal{C}$, then

$$
(I \otimes \mathcal{R E})\left(\sum_{i}\left|e_{i}\right\rangle\left\langle e_{i}|\otimes| e_{i}\right\rangle\left\langle e_{i}\right|\right)=\lambda \sum_{i}\left|e_{i}\right\rangle\left\langle e_{i}|\otimes| e_{i}\right\rangle\left\langle e_{i}\right|
$$

for some $\lambda \in \mathbb{C}$.

Proof. $1 \Rightarrow 2$ : We have

$$
\begin{aligned}
\mathcal{R} \mathcal{E} P_{\mathcal{C}} T P_{\mathcal{C}} & =\sum_{r, a} R_{r} A_{a} P_{\mathcal{C}} T P_{\mathcal{C}} R_{r}^{*} A_{a}^{*}=\sum_{r, a} \lambda_{r, a} P_{\mathcal{C}} T P_{\mathcal{C}} R_{r}^{*} A_{a}^{*} \\
& =\sum_{r, a} \lambda_{r, a} \bar{\lambda}_{r, a} P_{\mathcal{C}} T P_{\mathcal{C}}=\lambda P_{\mathcal{C}} T P_{\mathcal{C}}
\end{aligned}
$$

where $\lambda=\sum_{r, a}\left|\lambda_{r, a}\right|^{2}$.

$2 \Rightarrow 3$ : Note that since $\left|e_{i}\right\rangle \in \mathcal{C}$ we have $P_{\mathcal{C}}\left|e_{i}\right\rangle\left\langle e_{i}|=| e_{i}\right\rangle\left\langle e_{i}\right|$. Hence

$$
\begin{aligned}
(I \otimes \mathcal{R E})\left(\sum_{i}\left|e_{i}\right\rangle\left\langle e_{i}|\otimes| e_{i}\right\rangle\left\langle e_{i}\right|\right) & =\sum_{i}\left|e_{i}\right\rangle\left\langle e_{i}|\otimes \mathcal{R E}| e_{i}\right\rangle\left\langle e_{i}\right| \\
& =\sum_{i}\left|e_{i}\right\rangle\left\langle e_{i}\left|\otimes \mathcal{R E} P_{\mathcal{C}}\right| e_{i}\right\rangle\left\langle e_{i}\right| \\
& =\lambda \sum_{i}\left|e_{i}\right\rangle\left\langle e_{i}\left|\otimes P_{\mathcal{C}}\right| e_{i}\right\rangle\left\langle e_{i}\right| \\
& =\lambda \sum_{i}\left|e_{i}\right\rangle\left\langle e_{i}|\otimes| e_{i}\right\rangle\left\langle e_{i}\right|
\end{aligned}
$$


$3 \Rightarrow 1$ : Note that the Kraus operators for $\mathcal{R E}$ are $\left\{R_{r} A_{a}\right\}$. Therefore

$$
\begin{aligned}
\lambda \sum_{i}\left|e_{i}\right\rangle\left\langle e_{i}|\otimes| e_{i}\right\rangle\left\langle e_{i}\right| & =(I \otimes \mathcal{R E})\left(\sum_{i}\left|e_{i}\right\rangle\left\langle e_{i}|\otimes| e_{i}\right\rangle\left\langle e_{i}\right|\right) \\
& =\sum_{i, r, a}\left|e_{i}\right\rangle\left\langle e_{i}\left|\otimes R_{r} A_{a}\right| e_{i}\right\rangle\left\langle e_{i}\right| A_{a}^{*} R_{r}^{*}
\end{aligned}
$$

The result then follows since $\left|e_{i}\right\rangle\left\langle e_{i}\right|$ are linearly independent.

Definition 5.3.1. If the (equivalent) conditions of the above theorem are satisfied, we say the pair $(\mathcal{C}, \mathcal{R})$ is an $\mathcal{E}$-correcting code.

Next we discuss necessary and sufficient conditions for existence of a quantum error correcting code.

First, note that for a quantum code to correct the errors, it should distinguish the effects of two distinct errors applied to the same state of a quantum system. Since a quantum state is represented as a superposition of the basis states, a necessary condition for distinguishability is that orthogonal basis states be transformed to orthogonal states by the two distinct error operators. Second, an obvious requirement is that the measurement required by the error correction scheme should not modify the state of the system, i.e. should not reveal any information about the state of the system.

In fact, as the next theorem shows, these two obviously necessary condition are sufficient too.

Theorem 5.3.2. [17, Theorem III.2] A quantum code $\mathcal{C}$ can be extended to an $\mathcal{A}$ correcting code if and only if for any choice of an orthonormal basis $\left\{\left|e_{i}\right\rangle\right\}$ for $\mathcal{C}$, the following two conditions hold: 
(1) For every $\left|e_{i}\right\rangle$ and $\left|e_{j}\right\rangle$ with $i \neq j$ and operators $A_{a}, A_{b}$ in $\mathcal{A}$

$$
\left\langle e_{i}\left|A_{a}^{*} A_{b}\right| e_{j}\right\rangle=0 .
$$

(2) For every $\left|e_{i}\right\rangle$ and $\left|e_{j}\right\rangle$ with $i \neq j$ and operators $A_{a}, A_{b}$ in $\mathcal{A}$

$$
\left\langle e_{i}\left|A_{a}^{*} A_{b}\right| e_{i}\right\rangle=\left\langle e_{j}\left|A_{a}^{*} A_{b}\right| e_{j}\right\rangle .
$$

Proof. First suppose that $(\mathcal{C}, \mathcal{R})$ is an $\mathcal{A}$-correcting code. Then using Theorem 5.3.1 we obtain

$$
\begin{aligned}
\left\langle e_{i}\left|A_{a}^{*} A_{b}\right| e_{j}\right\rangle & =\left\langle e_{i}\left|A_{a}^{*} I A_{b}\right| e_{j}\right\rangle=\left\langle e_{i}\left|A_{a}^{*} \sum_{r} R_{r}^{*} R_{r} A_{b}\right| e_{j}\right\rangle \\
& =\sum_{r}\left\langle e_{i}\left|A_{a}^{*} R_{r}^{*} R_{r} A_{b}\right| e_{j}\right\rangle=\sum_{r}\left\langle e_{i}\left|\lambda_{a r}^{-} \lambda_{b r}\right| e_{j}\right\rangle \\
& =\alpha_{a b} \delta_{i j} .
\end{aligned}
$$

This implies both (1) and (2).

We now prove the reverse implication. Let $\mathcal{V}^{i}$ be the subspace spanned by $\left\{A_{a}\left|e_{i}\right\rangle\right\}_{a}$. Then it follows from (1) that the subspaces $\mathcal{V}^{i}$ are orthogonal. Let $\left|v_{r}^{i}\right\rangle$ be an orthonormal basis for $\mathcal{V}^{i}$. Since $\left|v_{r}^{i}\right\rangle$ 's are mutually orthogonal, there exists a unitary

$$
V_{r}\left|v_{r}^{i}\right\rangle=\left|e_{i}\right\rangle .
$$

Now let

$$
R_{r}:=V_{r} \sum_{i}\left|v_{r}^{i}\right\rangle\left\langle v_{r}^{i}\right|,
$$

and define a quantum channel $\mathcal{R}$ determined by the Kraus operators $\left\{\mathcal{P}^{\prime}, R_{1}, R_{2}, \ldots\right\}$, where $\mathcal{P}^{\prime}$ is the projection onto the orthogonal complement of $\oplus_{i} \mathcal{V}^{i}$. 
We show that $\mathcal{R}$ is in fact a recovery operator. First, note that $\mathcal{R}$ is a quantum channel, since it is a sum of orthogonal projections followed by unitary operators where the projections span the Hilbert space. It follows from (ii) that for a fixed $i_{0}$ there is a unitary $U_{i}$ for each $i$ such that $U_{i}\left|v_{r}^{i_{0}}\right\rangle=\left|v_{r}^{i}\right\rangle$, and $U_{i} A_{a}\left|e_{i_{0}}\right\rangle=A_{a}\left|e_{i}\right\rangle$ for all $a$. Then for each $A_{a}$, and $\psi \in \mathcal{C}$ there are scalars $\left\{\alpha_{i}\right\}_{i}$ and $\left\{\beta_{a r}^{i_{0}}\right\}_{r}$ such that

$$
\begin{aligned}
A_{a}|\psi\rangle & =A_{a} \sum_{i} \alpha_{i}\left|e_{i}\right\rangle=\sum_{i} \alpha_{i} A_{a}\left|e_{i}\right\rangle \\
& =\sum_{i} \alpha_{i} U_{i} A_{a}\left|e_{i_{0}}\right\rangle=\sum_{i, r} \alpha_{i} U_{i} \beta_{a r}^{i_{0}}\left|v_{r}^{i_{0}}\right\rangle \\
& =\sum_{i, r} \alpha_{i} \beta_{a r}^{i_{0}}\left|v_{r}^{i}\right\rangle .
\end{aligned}
$$

Therefore

$$
\begin{aligned}
R_{r} A_{a}|\psi\rangle & =\sum_{i} V_{r}\left|v_{r}^{i}\right\rangle\left\langle v_{r}^{i}\left|\sum_{j, s} \alpha_{j} \beta_{a s}^{i_{0}}\right| v_{s}^{j}\right\rangle=\sum_{i} \alpha_{i} \beta_{a r}^{i_{0}} V_{r}\left|v_{r}^{i}\right\rangle \\
& =\sum_{i} \beta_{a r}^{i_{0}} \alpha_{i}\left|e_{i}\right\rangle=\beta_{a r}^{i_{0}}|\psi\rangle .
\end{aligned}
$$

This implies that $R_{r} A_{a}$ is a multiple of the identity operation on $\mathcal{C}$, and hence the desired result follows from Theorem 5.3.1.

\subsection{Passive methods of quantum error correction}

In this section we consider a different approach to quantum error correction. Recall in the (active) methods introduced in the previous section, one first encodes the information in an appropriate way, then let them pass through the noisy channel and be affected by the possible errors. Then by performing appropriate measurements, the type and the location of errors are determined. The last step then would be to undo all the changes caused to the state by errors. 
A different approach to the problem of finding correcting codes is the following. First we identify possible errors, i.e. interaction operators (Kraus operators of the error channel). Then we search for a large enough subspace on which the interaction operators act trivially. All we need to do then is to use such a space to encode our information. In fact, this way, there will be no error on the information to be corrected!

\subsubsection{Decoherence-free subspaces}

Using representation theory tools, and under some physical conditions (which we will not address here), one can show that there is always a distinguished subspace of the quantum system, remaining intact with respect to the interaction of the system with the environment. In the following we give a description of this subspace.

Recall that the special linear group $S L(2)$ is the group of all complex $2 \times 2$ matrices with determinant one. This group acts canonically on the system of $n$ qubits, since there is a canonical representation $\pi$ of this group on the underlying Hilbert space $\mathcal{H}_{2^{n}}$ (the $n$-th tensor product of the identity representation). Decomposing into irreducible representation, the states corresponding to the trivial representation are called the singlet states. In other words, a singlet state is a state which remains fixed by every $\pi(g), g \in S L(2)$.

Example 5.4.1. Let us denote by $\pi_{j}$ the $(2 j+1)$-dimensional irreducible representation of $S L(2)$. Then $\pi_{0}$ and $\pi_{1 / 2}$ are, respectively, the trivial and the fundamental (i.e. the identity) representations. Then by the Clebsch-Gordan formula we get

$$
\begin{gathered}
\pi_{1 / 2}^{\otimes 2}=\pi_{0} \oplus \pi_{1} ; \\
\pi_{1 / 2}^{\otimes 4}=2 \pi_{0} \oplus 3 \pi_{1} \oplus \pi_{2} ;
\end{gathered}
$$




$$
\pi_{1 / 2}^{\otimes 6}=5 \pi_{0} \oplus 9 \pi_{1} \oplus 5 \pi_{2} \oplus \pi_{3}
$$

Hence, there are one singlet state for $n=2$, two singlet states for $n=4$, and five for $n=6$. In the case $n=2$ the one singlet state is the Bell state

$$
\left|\psi^{(2)}\right\rangle=\frac{1}{\sqrt{2}}(|01\rangle-|10\rangle)
$$

In the case $n=4$ one can choose as a basis in a two dimensional singlets space two (nonorthogonal) vectors

$$
\begin{aligned}
& \left|\psi_{1}^{(4)}\right\rangle=\frac{1}{2}(|1001\rangle-|0101\rangle+|0110\rangle-|1010\rangle), \\
& \left|\psi_{2}^{(4)}\right\rangle=\frac{1}{2}(|1001\rangle-|0011\rangle+|0110\rangle-|1100\rangle) .
\end{aligned}
$$

We denote by $\mathcal{C}_{n}$ the subspace spanned by the singlet states. Next theorem shows the space $\mathcal{C}_{n}$ can be used as a noiseless quantum code in which information can be stored without being affected by errors. This space is also called the decoherence free subspace.

The physical model of error caused on the state of a quantum system due to interaction by the environment is as follows. If the system is at initial state $\rho$, and the environment is at the initial state $\rho_{B}$, the the whole (composite) system is at the initial state $\rho \otimes \rho_{B}$. The state of the system at the time $t$ will then be given by $U(t)\left(\rho \otimes \rho_{B}\right) U^{*}(t)$, where $U(t)$ is unitary for all $t$.

Under some physical conditions, the unitaries $U(t)$ split as the tensor product $U_{1}(t) \otimes U_{2}(t)$, where $U_{1}(t)$ and $U_{2}(t)$ are unitaries on the quantum system and the environment, respectively.

Under such conditions we have: 
Theorem 5.4.1. [41, Theorem 1] Let $\mathcal{M}_{n}$ be the set of all states on the singlet space $\mathcal{C}_{n}$. If $\rho=\sum_{i j} \gamma_{i j}\left|\psi_{i}\right\rangle\left\langle\psi_{j}\right| \in \mathcal{M}_{n}$, then for any initial state $\rho_{B}$ of the environment we have $\operatorname{tr}_{B}\left(U(t)\left(\rho \otimes \rho_{B}\right) U^{*}(t)\right)=\rho$, for all $t>0$, where $U(t)$ is the unitary that implements the evolution of the system, and $\operatorname{tr}_{B}$ is the partial trace over the environment.

Proof. Let $\rho_{B}=\sum_{k, l} b_{k l}\left|e_{k}\right\rangle\left\langle e_{l}\right|$, and $\rho=\sum_{i j} \gamma_{i j}\left|\psi_{i}\right\rangle\left\langle\psi_{j}\right|$, then

$$
\begin{aligned}
U(t)\left(\rho \otimes \rho_{B}\right) U^{*}(t) & =\sum_{i, j, k, l} \gamma_{i j} b_{k l} U(t)\left(\left|\psi_{i}\right\rangle \otimes\left|e_{k}\right\rangle\right)\left(\left\langle\psi_{j} \otimes\left\langle e_{l}\right|\right) U^{*}(t)\right. \\
& =\sum_{i, j, k, l} \gamma_{i j} b_{k l}\left(U_{1}(t)\left|\psi_{i}\right\rangle \otimes U_{2}(t)\left|e_{k}\right\rangle\right)\left(\left\langle\psi_{j}\right| U_{1}^{*}(t) \otimes\left\langle e_{l}\right| U_{2}^{*}(t)\right) \\
& =\sum_{i, j, k, l} \gamma_{i j} b_{k l}\left(\left|\psi_{i}\right\rangle \otimes U_{2}(t)\left|e_{k}\right\rangle\right)\left(\left\langle\psi_{j}\right| \otimes\left\langle e_{l}\right| U_{2}^{*}(t)\right) .
\end{aligned}
$$

Now taking the partial trace over the environment, we obtain

$$
\begin{aligned}
\operatorname{tr}_{B}\left(U(t)\left(\rho \otimes \rho_{B}\right) U^{*}(t)\right) & =\left(\sum_{i j} \gamma_{i j}\left|\psi_{i}\right\rangle\left\langle\psi_{j}\right|\right)\left(\sum_{k l} b_{k l} \operatorname{tr}\left(\left|e_{k}\right\rangle\left\langle e_{l}\right|\right)\right) \\
& =\sum_{i j} \gamma_{i j}\left|\psi_{i}\right\rangle\left\langle\psi_{j}\right| \sum_{l} b_{l l} \\
& =\rho .
\end{aligned}
$$

\subsubsection{Noiseless subsystems}

In the previous section we presented a way of passive error correction by encoding our information into a subspace which is immune from the noise caused by the interaction with the environment. More precisely, the states on this subspace were remained fixed under the noise operator. Therefore, the space spanned by such states is a subspace 
of the fixed point space of the noise operator:

$$
\operatorname{Fix}(\mathcal{E}):=\{T \in \mathcal{B}(\mathcal{H}): \mathcal{E}(T)=T\}
$$

Given the noise operator $\mathcal{E}$, the main problem in passive methods of error correction is to concretely identify an immune subspace in order to encode the information. We saw in last section that with certain physical conditions, using representation theory, we can construct a concrete subspace of $\operatorname{Fix}(\mathcal{E})$, namely the singlet space.

Since those imposed physical condition do not necessarily hold for all systems, one should account for the cases in which they fail. To emphasize, the problem here is to find a concrete subspace of $\operatorname{Fix}(\mathcal{E})$ for a given noise operator $\mathcal{E}$.

As before, we denote by $\left\{A_{a}\right\}$ the Kraus operators of $\mathcal{E}$. We define the noise commutant $\mathcal{C}_{\mathcal{E}}$ of $\mathcal{E}$ as

$$
\mathcal{C}_{\mathcal{E}}:=\left\{A_{a}\right\}^{\prime}=\left\{T \in \mathcal{B}(\mathcal{H}): A_{a} T=T A_{a}, \forall a\right\}
$$

Observe that $\mathcal{C}_{\mathcal{E}}$ is a $C^{*}$-subalgebra (i.e. a self-adjoint subalgebra) of $\mathcal{B}(\mathcal{H})$. Moreover, if $\mathcal{E}$ is unital (such quantum channels are called bi-stochastic), then $C_{\mathcal{E}} \leq \operatorname{Fix}(\mathcal{E})$. Since the structure theory of (finite dimensional) $C^{*}$-subalgebras of $\mathcal{B}(\mathcal{H})$ are completely known (up to unitary equivalence), these observations suggest that in the case of unital noise operators, we may use operator algebra techniques to construct noiseless subspaces. In fact, next theorem, which is due to Kribs [20], shows that we can get our hands on the whole fixed point space of the noise operator. But first we need a lemma.

Lemma 5.4.1. [20, Lemma 2.3] Let $\Phi: \mathcal{B}(\mathcal{H}) \rightarrow \mathcal{B}(\mathcal{H})$ be a unital quantum channel with Kraus operators $\left\{A_{1}, \ldots, A_{n}\right\}$, and let $P$ be a projection on $\mathcal{H}$. If the subspace 
$P \mathcal{H}$ is invariant for $A_{1}, \ldots, A_{n}$, i.e. $P A_{k}=P A_{k} P, 1 \leq k \leq n$, then it reduces $A_{1}, \ldots, A_{n}$, i.e. $A_{k} P=P A_{k}, 1 \leq k \leq n$.

Proof. Since $P \mathcal{H}$ is invariant for $A_{1}, \ldots, A_{n}$, we get

$$
0 \leq \Phi(P)=\sum_{k} A_{k} P A_{k}^{*}=P \Phi(P) P \leq P \Phi(\mathbb{1}) P=P \mathbb{1} P=P
$$

Since moreover $\operatorname{tr}(P-\Phi(P))=0$, it follows that $\Phi(P)=P$. Therefore

$$
\Phi\left(P^{\perp}\right)=\Phi(1-P)=P^{\perp}
$$

Hence, for each $i$ we get

$$
0 \leq\left(P^{\perp} A_{i}^{*} P\right)^{*}\left(P^{\perp} A_{i}^{*} P\right) \leq \sum_{k}\left(P^{\perp} A_{k}^{*} P\right)^{*}\left(P^{\perp} A_{k}^{*} P\right)=P \Phi\left(P^{\perp}\right) P=0 .
$$

This implies $P^{\perp} A_{i}^{*} P=0$ for all $i$, and hence $\mathcal{P} \mathcal{H}$ is a reducing subspace for $A_{1}, \ldots, A_{n}$.

Theorem 5.4.2. [20, Theorem 2.1] Let $\Phi: \mathcal{B}(\mathcal{H}) \rightarrow \mathcal{B}(\mathcal{H})$ be a unital quantum channel with Kraus operators $\left\{A_{1}, \ldots, A_{n}\right\}$. Then the fixed point set for $\Phi$ is a $C^{*}$ algebra and

$$
\operatorname{Fix}(\Phi)=\left\{A_{1}, A_{2}, \ldots, A_{n}\right\}^{\prime}
$$

Proof. Let $\mathcal{A}$ denote the subalgebra of $\mathcal{B}(\mathcal{H})$ generated by the Kraus operators of $\Phi$, $\left\{A_{1}, \ldots, A_{n}\right\}$. The inclusion $\mathcal{A}^{\prime} \subseteq \operatorname{Fix}(\Phi)$ is obvious because $\Phi$ is unital. To see the converse, first observe that if $\operatorname{Fix}(\Phi)=\mathbb{C} 1$, then

$$
\mathcal{A}^{\prime} \subseteq \operatorname{Fix}(\Phi)=\mathbb{C} 1 \subseteq \mathcal{A}^{\prime}
$$


and the two sets coincide. Now, let $T \in \operatorname{Fix}(\Phi)$ be a non-scalar operator. Since $\operatorname{Fix}(\Phi)$ is self-ajoint and unital, it is generated by its positive elements, we may assume, without loss of generality, that $T \geq 0$. Let $0 \leq \lambda_{1}<\lambda_{2}<\ldots<\lambda_{r}$ be the eigenvalues of $T$, and $P_{1}, \ldots, P_{r}$ the corresponding spectral Projections. Then $T=\lambda_{1} P_{1}+\ldots+\lambda_{r} P_{r}$ by the Spectral Theorem. Let $\mathcal{H}_{r}$ denote the eigenspace of the eigenvalue $\lambda_{r}$. Let $\xi \in \mathcal{H}_{r}$ be a unit vector, then we have

$$
\begin{aligned}
\lambda_{r} & =\langle T \xi \| \xi\rangle=\sum_{k=1}^{n}\left\langle A_{k} T A_{k}^{*} \xi \| \xi\right\rangle=\sum_{k=1}^{n}\left\langle T A_{k}^{*} \xi \| A_{k}^{*} \xi\right\rangle \\
& \leq \lambda_{r} \sum_{k=1}^{n}\left\langle A_{k}^{*} \xi \| A_{k}^{*} \xi\right\rangle=\lambda_{r} \sum_{k=1}^{n}\left\langle A_{k} A_{k}^{*} \xi \| \xi\right\rangle \\
& =\lambda_{r} .
\end{aligned}
$$

This implies

$$
\sum_{k=1}^{n}\left\langle T A_{k}^{*} \xi \| A_{k}^{*} \xi\right\rangle=\lambda_{r} \sum_{k=1}^{n}\left\langle A_{k}^{*} \xi \| A_{k}^{*} \xi\right\rangle,
$$

and since $\left\langle T A_{k}^{*} \xi \| A_{k}^{*} \xi\right\rangle \leq \lambda_{r}\left\langle A_{k}^{*} \xi \| A_{k}^{*} \xi\right\rangle$ for all $1 \leq k \leq n$, it follows that $\left\langle T A_{k}^{*} \xi \| A_{k}^{*} \xi\right\rangle=\lambda_{r}\left\langle A_{k}^{*} \xi \| A_{k}^{*} \xi\right\rangle$ for all $1 \leq k \leq n$. Hence $A_{k}^{*} \xi \in \mathcal{H}_{r}$ for $k=1, \ldots, n$. This means $\mathcal{H}_{r}$ is an invariant subspace for $\mathcal{A}^{*}$, hence a reducing subspace for $\mathcal{A}$ by Lemma 5.4.1. Therefore, it follows that $P_{r} \in \mathcal{A}^{\prime}$. But then also $P_{r} \in \operatorname{Fix}(\Phi)$, and so is $T-\lambda_{r} P_{r}$. We can now repeat the above argument to show that $P_{k} \in \mathcal{A}^{\prime}$ for $k=1, \ldots, n$. Hence $T \in \mathcal{A}^{\prime}$.

In the following, we present a constructive algorithm, developed by Holbrook, Kribs, and Laflamme [14] to compute explicitly the noise commutant $\mathcal{A}^{\prime}$ for a given unital quantum channel, assuming that it has been computed as a linear span.

First, let us note that since $\mathcal{A}$ is $C^{*}$-subalgebra of the finite dimensional $\mathcal{B}(\mathcal{H})$, 
there exists a basis for $\mathcal{H}$ with respect to which $\mathcal{A}$ is of the form

$$
\mathcal{A}=\left(\mathcal{M}_{n_{1}} \otimes 1_{m_{1}}\right) \oplus \cdots \oplus\left(\mathcal{M}_{n_{d}} \otimes 1_{m_{d}}\right)
$$

for some integers $m_{k}, n_{k}, k=1, \ldots, d$. The projections in $\mathcal{A}$ which correspond to the identity operators on the blocks $\mathcal{M}_{n_{k}} \otimes 1_{m_{k}}$ in the decomposition (5.4.1) are called the minimal central projections of $\mathcal{A}$.

Definition 5.4.1. Let $\left\{P_{j}\right\}$ be the (unique) maximal family of non-zero mutually orthogonal minimal $\mathcal{A}$-reducing projections. A subset $\left\{Q_{k}\right\}_{k \in S}$ of this family is linked in $\mathcal{A}$ if the following two conditions hold.

1. The projection $\mathcal{Q}=\sum_{k \in \mathcal{S}} \mathcal{Q}_{k}$ belongs to $\mathcal{A}$.

2. If $\mathcal{S}_{1}$ is a proper subset of $\mathcal{S} ;$ then the projection $\sum_{k \in \mathcal{S}_{1}} \mathcal{Q}_{k}$ does not belong $\mathcal{A}$.

Note that only projections with the same rank can be linked. So, let us group them together, and let $\left\{P_{j}: j \in \mathcal{S}_{k}\right\}_{k}$ be the linked subsets of projections from $\left\{P_{j}\right\}$. Since $\sum_{j \in \mathcal{S}_{k}} P_{j} \in \mathcal{A} \cap \mathcal{A}^{\prime}$, it follows that $\mathcal{A}\left(\sum_{j \in \mathcal{S}_{k}} P_{j}\right)$ is a subalgebra of $\mathcal{A}$.

Once the $*$-algebra structure of $\mathcal{A}$ has been identified, we can easily deduce the *-algebra structure of $\mathcal{A}^{\prime}=\operatorname{Fix}(\Phi)$. If $\mathcal{A}$ is given by (5.4.1), then

$$
\operatorname{Fix}(\Phi)=\mathcal{A}^{\prime} \simeq \bigoplus_{k}\left(\mathcal{M}_{n_{k}} \otimes 1_{m_{k}}\right)^{\prime} \simeq \bigoplus_{k}\left(1_{n_{k}} \otimes \mathcal{M}_{m_{k}}\right)
$$

We first outline the steps of the algorithm, and then give the details in a number of result proven below. Recall that we assume the noise commutant $\mathcal{A}^{\prime}=\operatorname{Fix}(\Phi)=$ span $\left\{B_{1}, B_{2}, \ldots, B_{r}\right\}$ has been identified as a linear span. So, the algorithm will indeed concretely identify the algebra structure of $\mathcal{A}$, and hence of $\mathcal{A}^{\prime}$, from knowing the vector space structure of $\mathcal{A}^{\prime}$. 


\section{Steps of the Algorithm:}

(i) If $\mathcal{A}^{\prime} \neq \mathbb{C} 1$, then choose non-scalar $T=T^{*} \in \mathcal{A}^{\prime}$.

(ii) Find the spectral projections $P_{1}, \cdots, P_{s}$ of $T$.

(iii) For each $P=P_{j}$, compute $P B_{1} P, \cdots, P B_{r} P$. If $P B_{i} P \in \mathbb{C} P$ for all $i$, then $P \mathcal{A}^{\prime} P=\mathbb{C} P$ and $P$ is a (non-zero) minimal reducing projection for $\mathcal{A}$, and it belongs to the maximal family.

(iv) If for some $i, P B_{i} P \notin \mathbb{C} P$, then $P$ is not minimal $\mathcal{A}$-reducing and the compression channel $\Phi_{P}:=P \Phi P$ has non-scalar fixed points. Then repeat steps $(i)$, (ii) and (iii), perhaps a number of times, to find minimal $\mathcal{A}$-reducing mutually orthogonal projections supported on $P$.

(v) Given the family $\left\{P_{j}\right\}$ from Part 1, group together those projections with the same rank.

(vi) For the projections $\left\{P_{j, k}\right\}_{j \in \mathcal{S}_{k}}$ of rank $k \geq 2$, test for links between the $P_{j, k}$ by evaluating the commutators $\left[P_{\mathcal{S}}, B_{i}\right]$ where $P_{\mathcal{S}}=\sum_{j \in \mathcal{S}} P_{j, k}$ with $\mathcal{S} \subseteq \mathcal{S}_{k}$. Then $\left\{P_{j, k}: k \in \mathcal{S}\right\}$ are linked in $\mathcal{A}$ if and only if $\left[P_{\mathcal{S}}, B_{i}\right]=0$ for each $i$; and there is no proper subset of $\mathcal{S}$ with this property. The projections $P_{\mathcal{S}}$ defined by linked sets of projections will form the family of minimal central projections for $\mathcal{A}$.

(vii) The various links will give the $*$-algebra structure of $\mathcal{A}$ up to unitary equivalence, and hence of $\mathcal{A}^{\prime}=\operatorname{Fix}(\Phi)$; along with the precise locations of matrix blocks inside $\mathcal{A}^{\prime}$.

In the rest of this section we give mathematical details of these steps.

Lemma 5.4.2. [14, Lemma III.4] The following are equivalent for an operator $T=T^{*}$ in $\mathcal{B}(\mathcal{H})$ : 
(i) $\Phi(T)=T$.

(ii) Every spectral projection $P$ of $T$ satisfies $\Phi(P)=P$.

(iii) Every spectral projection $P$ of $T$ belongs to $\mathcal{A}^{\prime}$.

Proof. The implications $(i i i) \Rightarrow(i i)$ and $(i i) \Rightarrow(i)$ are immediate. Now suppose $(i)$ holds. Then by Theorem 5.4.2 $T \in \mathcal{A}^{\prime}$, and since every spectral projection of $T$ is in the $C^{*}$-algebra generated by $T$ (note that we are in finite-dimensional case), (iii) follows.

The following lemma is a direct consequence of Theorem 5.4.2.

Lemma 5.4.3. [14, Lemma IV.3] Let $\Psi: \mathcal{B}(\mathcal{H}) \rightarrow \mathcal{B}(\mathcal{H})$ be a unital quantum quantum channel, with the Kraus operators $C_{1}, \cdots, C_{m}$, and let $\mathcal{C}=\operatorname{Alg}\left\{C_{1}, \cdots, C_{m}\right\}$. Then the following are equivalent:

(i) $\operatorname{Fix}(\Psi)=\mathcal{C}^{\prime}=\mathbb{C} 1$.

(ii) There are no non-trivial reducing subspaces for the algebra $\mathcal{C}$.

Proposition 5.4.1. With the assumption of Lemma 5.4.3, if $P \in \mathcal{B}(\mathcal{H})$ is a projection that reduces $\mathcal{C}$, then the unital channel $\Psi_{P}: \mathcal{B}(\mathcal{H}) \rightarrow \mathcal{B}(\mathcal{H})$ given by

$$
\Psi_{P}(T)=\sum_{i} C_{i, P} T C_{i, P}^{*}
$$

where $C_{i, P}=C_{i} P=P C_{i}$, satisfies

$$
\operatorname{Fix}\left(\Psi_{P}\right)=\left.P \mathcal{C}^{\prime}\right|_{P \mathcal{H}}=\left(\left.\mathcal{C}\right|_{P \mathcal{H}}\right)^{\prime}=\left(\left.P \mathcal{C}\right|_{P \mathcal{H}}\right)^{\prime}
$$

Furthermore, $P$ is a minimal projection in $\mathcal{C}^{\prime}$ if and only if

$$
\operatorname{Fix}\left(\Psi_{P}\right)=P \mathcal{C}^{\prime} P=\mathbb{C} P .
$$


Proof. Let $T \in \mathcal{C}^{\prime}$. Then

$$
(P T P)\left(C_{i} P\right)=P\left(T C_{i}\right) P=P\left(C_{i} T\right) P=\left(P C_{i} P\right)(P T P)=\left(C_{i} P\right)(P T P),
$$

which implies $P \mathcal{C}^{\prime} P \subseteq(\mathcal{C} P)^{\prime}$. Conversely, if $T=P T P$ is in $(\mathcal{C} P)^{\prime}$, then

$$
T C_{i}=T P C_{i}=T\left(C_{i} P\right)=\left(C_{i} P\right) T=C_{i} T,
$$

and therefore $T \in P \mathcal{C}^{\prime} P$. Hence, by Theorem 5.4.2

$$
\operatorname{Fix}\left(\Psi_{P}\right)=\left\{C_{i P}\right\}^{\prime}=\left\{C_{i} P\right\}^{\prime}=(\mathcal{C} P)^{\prime}=P \mathcal{C}^{\prime} P .
$$

Other implications follow from Lemma 5.4.3.

If we apply this result to $P=P_{j}$, it implies $P_{j}$ is a minimal projection in $\mathcal{A}^{\prime}$ if and only if $P_{j} \mathcal{A}^{\prime} P_{j}=C P_{j}$. Since we are assuming the noise commutant $\mathcal{A}^{\prime}$ is known as a linear span, we can then simply compute $P_{j} B P_{j}$ for a set of vector space generators $B$ for $\mathcal{A}^{\prime}$, and check whether each $P_{j} B P_{j}$ is in $\mathbb{C} P_{j}$. If this is the case, then $P_{j}$ is minimal and it belongs to the maximal family of projections we need. On the other hand, if there is a $B_{0}$ in $\mathcal{A}^{\prime}$ such that $P_{j} B_{0} P_{j}$ is not in $\mathbb{C} P_{j}$, then $P_{j}$ is not minimal. In this case we repeat the above process. Indeed, $\Phi_{j}$ is a unital channel on $\mathcal{B}\left(P_{j} \mathcal{H}\right)$ with non-scalar fixed points, in fact $P_{j} B_{0} P_{j} \in \mathcal{A}^{\prime} P_{j}=\operatorname{Fix}\left(\Phi_{j}\right)$ is such an operator. Thus, we may use $P_{j} B_{0} P_{j}$ to obtain a self-adjoint operator in $\operatorname{Fix}\left(\Phi_{j}\right)$, and continue the process by considering the spectral projections of this operator, which are subprojections of $P_{j}$. As $\mathcal{H}$ is finite dimensional, this process can have finitely many steps. We will be left with the maximal family (since they sum to the identity operator) of mutually orthogonal non-zero minimal projections in $\mathcal{A}^{\prime}$.

For $k \geq 1$, let $\left\{P_{j, k}\right\}$ be the projections amongst $\left\{P_{j}\right\}$ with $\operatorname{dim} P_{j, k} \mathcal{H}=k$. Further 
let $\mathcal{S}_{k}$ be the index set for this set of projections. Suppose $\mathcal{A}^{\prime}=\operatorname{span}\left\{B_{1}, \cdots, B_{r}\right\}$ with $B_{i}$ in $\mathcal{B}(\mathcal{H})$. Since $\mathcal{A}=\left(\mathcal{A}^{\prime}\right)^{\prime}=\left(\operatorname{span}\left\{B_{1}, \cdots, B_{r}\right\}\right)^{\prime}$, we may check if projections of the form $P_{\mathcal{S}}=\sum_{j \in \mathcal{S}} P_{j, k}$, where $\mathcal{S} \subseteq \mathcal{S}_{k}$, belong to $\mathcal{A}$, simply by computing the commutators

$$
\left[P_{\mathcal{S}}, B_{i}\right]=P_{\mathcal{S}} B_{i}-B_{i} P_{\mathcal{S}} \quad \text { for } \quad i=1, \ldots, r
$$

The projections $\left\{P_{j, k}\right\}_{j \in \mathcal{S}}$ are linked in $\mathcal{A}$ precisely when $\left[P_{\mathcal{S}}, B_{i}\right]=0$ for each $i$, and, there is no proper subset of $\mathcal{S}$ with this property. As $\mathcal{H}$ is finite dimensional, we can find the links between the projections $\left\{P_{j, k}\right\}$, after only finitely many computations.

\subsection{Operator quantum error correction}

In this last section of the thesis, we present a method of quantum error correction that generalizes, and unifies all previously introduced methods. This scheme, called Operator Quantum Error Correction was introduced by Kribs, Laflamme, and Poulin [21].

In this unified scheme for quantum error correction we consider a Hilbert space $\mathcal{H}$ with a fixed decomposition $\mathcal{H}=\left(\mathcal{H}^{A} \otimes \mathcal{H}^{B}\right) \oplus \mathcal{K}$, and we deal with a noise operator $\mathcal{E}$. We also denote by $\mathfrak{A}$ the semigroup of operators in $T \in \mathcal{B}(\mathcal{H})$ that are of the form $T=T^{A} \otimes T^{B}$ for some $T^{A} \in \mathcal{B}\left(\mathcal{H}^{A}\right)$ and $T^{B} \in \mathcal{B}\left(\mathcal{H}^{B}\right)$, and we let $\mathfrak{A}_{0}$ be the subsemigroup of operators of the form $T=\mathbb{1}^{A} \otimes T^{B}$ for some $T^{B} \in \mathcal{B}\left(\mathcal{H}^{B}\right)$. Also, $\mathcal{P}_{\mathfrak{A}}$ denotes the mapping $\mathcal{B}(\mathcal{H}) \ni T \mapsto P_{T^{A} \otimes T^{B}} T P_{T^{A} \otimes T^{B}}$.

Definition 5.5.1. We say $\mathfrak{A}$ is correctable for $\mathcal{E}$ if there is a quantum channel $\mathcal{R}$ on $\mathcal{H}$ such that

$$
\left(\operatorname{tr}_{A} \circ \mathcal{P}_{\mathfrak{A}} \circ \mathcal{R} \circ \mathcal{E}\right)(T)=\operatorname{tr}_{A}(T) \quad \text { for all } T \in \mathfrak{A}
$$

Note that if $\mathfrak{A}$ is correctable for $\mathcal{E}$, then $\mathcal{H}^{B}$ provides a noiseless subsystem. 
In the following result we give more equivalent conditions for the semigroup $\mathfrak{A}$ to be correctable for $\mathcal{E}$.

Theorem 5.5.1. [22] Let $\mathcal{H}, \mathcal{E}$, and $\mathfrak{A}$ be as above. The following conditions are equivalent:

1. $\mathfrak{A}$ is correctable for $\mathcal{E}$.

2. There is a quantum channel $\mathcal{R}$ on $\mathcal{H}$ such that for every $T^{A} \in \mathcal{B}\left(\mathcal{H}^{A}\right)$ and $T^{B} \in \mathcal{B}\left(\mathcal{H}^{B}\right)$ there is $S^{A} \in \mathcal{B}\left(\mathcal{H}^{A}\right)$ such that

$$
\mathcal{R} \circ \mathcal{E}\left(T^{A} \otimes T^{B}\right)=S^{A} \otimes T^{B}
$$

3. There is a quantum channel $\mathcal{R}$ on $\mathcal{H}$ such that for every $T^{B} \in \mathcal{B}\left(\mathcal{H}^{B}\right)$ there is $S^{A} \in \mathcal{B}\left(\mathcal{H}^{A}\right)$ such that

$$
\mathcal{R} \circ \mathcal{E}\left(\mathbb{1}^{A} \otimes T^{B}\right)=S^{A} \otimes T^{B}
$$

4. There is a quantum channel $\mathcal{R}$ on $\mathcal{H}$ such that for every $T \in \mathfrak{A}_{0}$

$$
(\mathcal{R} \circ \mathcal{E})(T)=T
$$

5. If $\left\{\left|\alpha_{k}\right\rangle\right\}$ is an orthonormal basis for $\mathcal{H}^{A}$, and $P_{k l}=\left|\alpha_{k}\right\rangle\left\langle\alpha_{l}\right| \otimes \mathbb{1}^{B}$, then there are scalars $\lambda_{a b k l}$ such that

$$
P_{k k} E_{a}^{*} E_{b} P_{l l}=\lambda_{a b k l} P_{k l}
$$

for all $a, b, k$, and $l$. 
Proof. The implications (2) $\Rightarrow(1),(2) \Rightarrow(3)$, and (4) $\Rightarrow(3)$ are trivial.

To prove $(3) \Rightarrow(2)$, choose $|\beta\rangle \in \mathcal{H}^{B}$, and let $P=|\beta\rangle\langle\beta|$. Also, let $\left\{\left|\alpha_{k}\right\rangle\right\}$ be an orthonormal basis for $\mathcal{H}^{A}$. Then

$$
0 \leq \mathcal{R} \circ \mathcal{E}\left(\left|\alpha_{k}\right\rangle\left\langle\alpha_{k}\right| \otimes P\right) \leq \mathcal{R} \circ \mathcal{E}\left(\mathbb{1}^{A} \otimes P\right)=S^{A} \otimes P .
$$

Therefore, for every $0 \leq T^{A} \in \mathcal{B}\left(\mathcal{H}^{A}\right)$ we have

$$
\operatorname{tr}_{A}\left(\left(T^{A} \otimes \mathbb{1}^{B}\right) \mathcal{R} \circ \mathcal{E}\left(\left|\alpha_{k}\right\rangle\left\langle\alpha_{k}\right| \otimes P\right) \leq \operatorname{tr}_{A}\left(\left(T^{A} \otimes \mathbb{1}^{B}\right)\left(S^{A} \otimes P\right)\right)=\operatorname{tr}\left(T^{A} S^{A}\right) P .\right.
$$

But since $P$ is a rank one projection, $\operatorname{tr}_{A}\left(\left(T^{A} \otimes \mathbb{1}^{B}\right) \mathcal{R} \circ \mathcal{E}\left(\left|\alpha_{k}\right\rangle\left\langle\alpha_{k}\right| \otimes P\right)\right) \in \mathbb{C} P$. The latter holds for all $T^{A} \in \mathcal{B}\left(\mathcal{H}^{A}\right)$, by linearity. Hence, there is an operator $S_{k}^{A} \in \mathcal{B}\left(\mathcal{H}^{A}\right)$ such that

$$
\mathcal{R} \circ \mathcal{E}\left(\left|\alpha_{k}\right\rangle\left\langle\alpha_{k}\right| \otimes P\right)=S_{k}^{A} \otimes P
$$

The operators $S_{k}^{A}$ do not depend on $|\beta\rangle$, and so (2) follows from the linearity of $\mathcal{R} \circ \mathcal{E}$. $(1) \Rightarrow(3)$ : it follows that

$$
\mathcal{P}_{\mathfrak{A}} \circ \mathcal{R} \circ \mathcal{E}(T)=\mathcal{R} \circ \mathcal{E}(T)
$$

for all $T \in \mathfrak{A}$. Similarly to the argument of the last implication above, we choose $|\beta\rangle \in \mathcal{H}^{B}$ and let $P=|\beta\rangle\langle\beta|$. Then we have

$$
\operatorname{tr}_{A}\left(\left(\mathbb{1}^{A} \otimes P\right)\left(\mathcal{R} \circ \mathcal{E}\left(\mathbb{1}^{A} \otimes P\right)\right)\left(\mathbb{1}^{A} \otimes P\right)\right)=\operatorname{tr}_{A}\left(\mathbb{1}^{A} \otimes P\right)=P .
$$

Now a similar argument as in the last implications implies that there exists $S^{A}$, independent of $|\Psi\rangle$, such that $\mathcal{R} \circ \mathcal{E}\left(\mathbb{1}^{A} \otimes P\right)=S^{A} \otimes P$, and therefore (3) follows. $(3) \Rightarrow(4)$ : Let $\left\{\left|\alpha_{k}\right\rangle\right\}$ be an orthonormal basis for $\mathcal{H}^{A}$, and let $P_{k l}=\left|\alpha_{k}\right\rangle\left\langle\alpha_{l}\right| \otimes \mathbb{1}^{B}$. 
Let $\Psi$ be the quantum channel on $\mathcal{B}\left(\mathcal{H}^{A} \otimes \mathcal{H}^{B}\right)$ determined by the Kraus operators $\left\{\frac{1}{\sqrt{m}} P_{k l}\right\}$. A straightforward calculation yields

$$
\Psi(T)=\sum_{k, l} P_{k l} T P_{k l}^{*}=\mathbb{1}^{A} \otimes\left(\operatorname{tr}_{A} \circ \mathcal{P}_{\mathfrak{A}}\right)(T) \in \mathbb{1}^{A} \otimes \mathcal{B}\left(\mathcal{H}^{B}\right)
$$

for all $T \in \mathcal{B}(\mathcal{H})$. Now suppose $\mathcal{R}^{\prime}$ be the channel given by (3). Then

$$
\left(\Psi \circ \mathcal{R}^{\prime} \circ \mathcal{E}\right)\left(\mathbb{1}^{A} \otimes T^{B}\right)=\Psi\left(S^{A} \otimes T^{B}\right)=\mathbb{1}^{A} \otimes T^{B}
$$

The map $\Psi$ can be extended to a quantum channel on $\mathcal{B}(\mathcal{H})$ by adding the projection $P_{\mathfrak{A}}^{\perp}$ onto $\mathcal{K}$ as a Kraus operator. As this does not effect (5.5.5), the result follows. $(4) \Rightarrow(5)$ : Let us first suppose that $\mathcal{R}$ is the identity channel. Let $\mathcal{C}=P_{\mathfrak{A}} \mathcal{H}$ be the range of the projection $P_{\mathfrak{A}}=P_{11}+\ldots+P_{m m}$. Then since $P_{\mathfrak{A}} \in \mathfrak{A}_{0}$ we have $\mathcal{E}\left(P_{\mathfrak{A}}\right)=P \mathfrak{A}$ and so $P_{\mathfrak{A}} E_{a \mid \mathcal{C}}=E_{a \mid \mathcal{C}}$ for all $a$ by Lemma 5.4.1. Define the completely positive $\operatorname{map} \mathcal{E}_{\mathcal{C}}: \mathcal{B}(\mathcal{C}) \rightarrow \mathcal{B}(\mathcal{C})$ by

$$
\mathcal{E}_{\mathcal{C}}(T)=\left.P_{\mathfrak{A}} \mathcal{E}(T)\right|_{\mathcal{C}}=\left.P_{\mathfrak{A}} \mathcal{E}\left(P_{\mathfrak{A}} T P_{\mathfrak{A}}\right)\right|_{\mathcal{C}} \quad(T \in \mathcal{B}(\mathcal{C}))
$$

Then we have

$$
\sum_{a}\left(\left.P_{\mathfrak{A}} E_{a}\right|_{\mathcal{C}}\right)^{*}\left(\left.P_{\mathfrak{A}} E_{a}\right|_{\mathcal{C}}\right)=\left.\sum_{a} P_{\mathfrak{A}} E_{a}^{*} E_{a}\right|_{\mathcal{C}}=P_{\mathfrak{A}} \mathbb{1}_{\mathcal{H} \mid \mathcal{C}}=\mathbb{1}_{\mathcal{C}}
$$

which shows that $\mathcal{E}_{\mathcal{C}}$ is indeed a quantum channel on $\mathcal{B}(\mathcal{C})$. Moreover, $\mathcal{E}_{\mathcal{C}}$ is unital as $\mathcal{E}_{\mathcal{C}}\left(\mathbb{1}_{\mathcal{C}}\right)=\left.P_{\mathfrak{A}} \mathcal{E}\left(P_{\mathfrak{A}}\right)\right|_{\mathcal{C}}=\left.\mathbb{1}\right|_{\mathcal{C}}$. Hence it follows from Theorem 5.4.2 that

$$
\mathfrak{A}_{0 \mid \mathcal{C}} \subseteq \operatorname{Fix}\left(\mathcal{E}_{\mathcal{C}}\right)=\left\{P_{\mathfrak{A}} E_{a \mid \mathcal{C}}, P_{\mathfrak{A}} E_{a \mid \mathcal{C}}^{*}\right\}^{\prime}
$$


where the latter commutant is computed inside $\mathcal{B}(\mathcal{C})$. It follows that

$$
\mathcal{B}\left(\mathcal{H}^{A}\right) \otimes \mathbb{1}^{B}=\left(\left.\mathfrak{A}_{0}\right|_{\mathcal{C}}\right)^{\prime} \supseteq\left\{\left.P_{\mathfrak{A}} E_{a}\right|_{\mathcal{C}},\left.P_{\mathfrak{A}} E_{a}^{*}\right|_{\mathcal{C}}\right\}^{\prime \prime}=C^{*}\left(\left\{\left.P_{\mathfrak{A}} E_{a}\right|_{\mathcal{C}}\right\}\right) .
$$

Since $P_{k l}$ generate $\left(\left.P_{\mathfrak{A l}} \mathfrak{A}_{0}\right|_{\mathcal{C}}\right)^{\prime}=\mathcal{B}\left(\mathcal{H}^{A}\right) \otimes \mathbb{1}^{B}$ (as a vector space), there are scalars $\mu_{a k l} \in \mathbb{C}$ such that

$$
P_{k k} E_{a} P_{l l}=P_{k k}\left(\left.P_{\mathfrak{A}} E_{a}\right|_{\mathcal{C}}\right) P_{l l}=\mu_{a k l} P_{k l}
$$

So, let us now consider the general case. Let $\left\{R_{b}\right\}$ be the Kraus operators of $\mathcal{R}$. Then the Karus operators of the composition channel $\mathcal{R} \circ \mathcal{E}$ are $\left\{R_{b} E_{a}\right\}$. Hence by the above there are scalars $\mu_{a b k l}$ such that

$$
P_{k k} R_{b} E_{a} P_{l l}=\mu_{a b k l} P_{k l}
$$

for all $a, b, k$, and $l$. Then we have

$\left(P_{k k} R_{b} E_{a} P_{l l}\right)^{*}\left(P_{k^{\prime} k^{\prime}} R_{b} E_{a^{\prime}} P_{l^{\prime} l^{\prime}}\right)=\left(\overline{\mu_{a b k l}} P_{l k}\right)\left(\mu_{a^{\prime} b k^{\prime} l^{\prime}} P_{k^{\prime} l^{\prime}}\right)=\left\{\begin{array}{cl}\left(\overline{\mu_{a b k l}} \mu_{a^{\prime} b k l^{\prime}}\right) P_{l l^{\prime}} & \text { if } k=k^{\prime}, \\ 0 & \text { if } k \neq k^{\prime} .\end{array}\right.$

By Lemma 5.4.1 the the Kraus operators $R_{b} E_{a}$ leave $\mathcal{C}=P_{\mathfrak{A}} \mathcal{H}$ invariant. Therefore for fixed $a, a^{\prime}, l$, and $l^{\prime}$ we have

$$
\begin{aligned}
\overline{\mu_{a b k l}} \mu_{a^{\prime} b k l^{\prime}} P_{l l^{\prime}} & =\sum_{b, k}\left(P_{l l} E_{a}^{*} R_{b}^{*} P_{k k}\right)\left(P_{k k} R_{b} E_{a^{\prime}} P_{l^{\prime} l^{\prime}}\right) \\
& =\sum_{b} P_{l l} E_{a}^{*} R_{b}^{*} P_{\mathfrak{A}} R_{b} E_{a^{\prime}} P_{l^{\prime} l^{\prime}} \\
& =P_{l l} E_{a}^{*}\left(\sum_{b} R_{b}^{*} R_{b}\right) E_{a^{\prime}} P_{l^{\prime} l^{\prime}} \\
& =P_{l l} E_{a}^{*} E_{a^{\prime}} P_{l^{\prime} l^{\prime}} .
\end{aligned}
$$


Now to conclude the proof, we let

$$
\lambda_{a a^{\prime} l l^{\prime}}=\sum_{b, k} \overline{\mu_{a b k l}} \mu_{a^{\prime} b k l^{\prime}}
$$

for all $a, a^{\prime}, l$, and $l^{\prime}$.

$(5) \Rightarrow(4)$ : Choose an orthonormal basis $\left|\alpha_{k}\right\rangle$ for $\mathcal{H}^{A}$. Let $P_{k}=\left|\alpha_{k}\right\rangle\left\langle\alpha_{k}\right| \in \mathcal{B}\left(\mathcal{H}^{A}\right)$, and define a quantum channel $\mathcal{E}_{k}: \mathcal{B}\left(\mathcal{H}^{B}\right) \rightarrow \mathcal{B}(\mathcal{H})$ by

$$
\mathcal{E}_{k}\left(T^{B}\right)=\mathcal{E}\left(P_{k} \otimes T^{B}\right)
$$

If $\left\{E_{a}\right\}$ are the Kraus operators of $\mathcal{E}$, then

$$
\mathcal{E}_{k}\left(T^{B}\right)=\sum_{a} E_{a}\left(\left|\alpha_{k}\right\rangle\left\langle\alpha_{k}\right| \otimes T^{B}\right) E_{a}^{*} .
$$

We may therefore consider $\left\{E_{a, k}\right\}_{a}$ as the Kraus operators of $\mathcal{E}_{k}$, where $E_{a, k}=$ $E_{a} P_{\mathfrak{A}}\left|\alpha_{k}\right\rangle$. Now let $\mathcal{E}_{B}: \mathcal{B}\left(\mathcal{H}^{B}\right) \rightarrow \mathcal{B}(\mathcal{H})$ be the quantum channel defined by the Kraus operators $\left\{\frac{1}{\sqrt{m}} E_{a, k}\right\}_{a, k}$, where $m=\operatorname{dim}\left(\mathcal{H}^{A}\right)$. Then using (5.5.3) we obtain

$$
\begin{aligned}
\mathbb{1}^{B} E_{a, k}^{*} E_{b, l} \mathbb{1}^{B} & =\mathbb{1}^{B}\left\langle\alpha_{k}\left|P_{\mathfrak{A}} E_{a}^{*} E_{b} P_{\mathfrak{A}}\right| \alpha_{l}\right\rangle \mathbb{1}^{B} \\
& =\sum_{k^{\prime}, l^{\prime}} \mathbb{1}^{B}\left\langle\alpha_{k}\left|P_{k^{\prime} k^{\prime}} E_{a}^{*} E_{b} P_{l^{\prime} l^{\prime}}\right| \alpha_{l}\right\rangle \mathbb{1}^{B} \\
& =\sum_{k^{\prime}, l^{\prime}} \lambda_{a b k^{\prime} l^{\prime}} \mathbb{1}^{B}\left\langle\alpha_{k} \mid P_{k^{\prime} l^{\prime} \mid} \alpha_{l}\right\rangle \mathbb{1}^{B} \\
& =\lambda_{a b k l} \mathbb{1}^{B} .
\end{aligned}
$$

Hence, by Theorem 5.3.1 there is a quantum channel $\mathcal{R}: \mathcal{B}(\mathcal{H}) \rightarrow \mathcal{B}\left(\mathcal{H}^{B}\right)$ such that 
$(\mathcal{R} \circ \mathcal{E} \circ \mathcal{B})\left(T^{B}\right)=T^{B}$ for all $T^{B} \in \mathcal{B}\left(\mathcal{H}^{B}\right)$. Therefore

$$
\begin{aligned}
(\mathcal{R} \circ \mathcal{E})\left(\mathbb{1}^{A} \otimes T^{B}\right) & =\mathcal{R}\left(\sum_{k} \mathcal{E}_{k}\left(\rho^{B}\right)\right) \\
& =m \mathcal{R}\left(\sum_{k, a} \frac{1}{m} E_{a, k} T^{B} E_{a, k}^{*}\right) \\
& =m \mathcal{R} \circ \mathcal{E}_{B}\left(T^{B}\right) \\
& =m T^{B} .
\end{aligned}
$$

Remark 5.5.1. Comparing Theorem 5.5.1 with Theorem 5.3.1, we see that the standard model for error correction is a special case of the operator quantum error correction, which is realized when in the above model $\operatorname{dim}\left(\mathcal{H}^{A}\right)=1$.

Moreover, both the decoherence-free subspace and noiseless subsystem methods, which have been introduced in this chapter are also special cases of the operator quantum error correction method. They can be realized in the above model when $\mathcal{R}$ is the identity channel: the case $\operatorname{dim}\left(\mathcal{H}^{A}\right)=1$ for the decoherence-free subspace method, and $\operatorname{dim}\left(\mathcal{H}^{A}\right) \geq 1$ for the noiseless subsystem method. 


\section{Bibliography}

[1] D. Aharonov, and M. Ben-Or, Fault-tolerant quantum computation with constant error, In Proceedings of the twenty-ninth annual ACM symposium on Theory of computing, pp. 176-188. ACM, 1997.

[2] A. Ambainis, A. Smith, and K. Yang, Extracting quantum entanglement (general entanglement purification protocols), Computational Complexity, 2002. Proceedings. 17th IEEE Annual Conference on. IEEE, 2002.

[3] H. Barnum, M. A. Nielsen, and B. Schumacher, Information transmission through a noisy quantum channel, Phys. Rev. A 57, no. 6 (1998), 4153-4175.

[4] C. H. Bennett, D. P. DiVincenzo, J. A. Smolin, and W. K. Wootters, Mixed-state entanglement and quantum error correction, Phys. Rev. A 54, no. 5 (1996), 3824-3851.

[5] P. Blanchard, Decoherence: Theoretical, Experimental, and Conceptual Problems, Proceedings of a Workshop Held at Bielefeld, Germany, 10-14 Novbember 1998. Vol. 538. Springer, 2000.

[6] A. R. Calderbank and P. W. Shor, Good quantum error-correcting codes exist, Phys. Rev. A 54, no. 2 (1996), 1098-1106.

[7] J. Chiaverini, D. Leibfried, T. Schaetz, M. D. Barrett, R. B. Blakestad, J. Britton, W. M. Itano et al. Realization of quantum error correction, Nature 432, no. 7017 (2004), 602-605.

[8] D. G. Cory, M. D. Price, W. Maas, E. Knill, R. Laflamme, W. H. Zurek, T. F. Havel, and S. S. Somaroo, Experimental quantum error correction, Phys. Rev. Lett. 81, no. 10 (1998), $2152-2155$.

[9] T. M. Cover, and J. A. Thomas, Elements of information theory, John Wiley and Sons, 2012. 
[10] S. J. Devitt, , W. J. Munro, and K. Nemoto, Quantum error correction for beginners, Reports on Progress in Phys. 76, no. 7 (2013), 076001.

[11] D. P. DiVincenzo, and P. W. Shor, Fault-tolerant error correction with efficient quantum codes, Phys. rev. lett. 77, no. 15 (1996), 3260-3263.

[12] D. Gottesman, An introduction to quantum error correction and fault-tolerant quantum computation, Quantum Information Science and Its Contributions to Mathematics, Proceedings of Symposia in Applied Mathematics. Vol. 68. 2009.

[13] R. B. Griths, Quantum Channels, Kraus Operators, POVMs, Quantum Computation and Quantum Information Theory Course Notes, Carnegie Mellon University (2010).

[14] J. A. Holbrook, D. W. Kribs, and Raymond Laflamme, Noiseless subsystems and the structure of the commutant in quantum error correction, Quantum Information Processing 2 (2003), no. $5,381-419$.

[15] A. Y. Kitaev, Quantum computations: algorithms and error correction, Russian Mathematical Surveys 52, no. 6 (1997), 1191-1249.

[16] E. Knill, Quantum computing with realistically noisy devices, Nature 434 (2005), no. 7029, $39-44$.

[17] E. Knill, and R. Laflamme, Theory of quantum error-correcting codes, Phys. Rev. A 55 (1997), no. 2,900 .

[18] E. Knill, R. Laflamme, A. Ashikhmin, H. Barnum, L. Viola, and W. H. Zurek, Introduction to quantum error correction, arXiv preprint quant-ph/0207170 (2002).

[19] K. Kraus, States, effects and operations, Springer, 1983.

[20] D. W. Kribs, Quantum channels, wavelets, dilations and representations of $\mathcal{O}_{n}$, Proc. Edinb. Math. Soc. (2) 46 (2003), no. 2, 421-433.

[21] D. W. Kribs, R. Laflamme, and D. Poulin, Unified and generalized approach to quantum error correction, Physical review letters 94 (2005), no. 18, 180501.

[22] D. W. Kribs, R. Laflamme, D. Poulin, and Maia Lesosky, Operator quantum error correction, Quantum Information \& Computation 6 (2006), no. 4, 382-399. 
[23] D. A. Lidar, I. L. Chuang, and K. B. Whaley, Decoherence-free subspaces for quantum computation, Physical Review Letters 81 (1998), no.12, 2594-2597.

[24] H. Lo, S. Popescu, and T. Spiller, Introduction to Quantum Computation Information, World Scientific Publishing Co., Inc., 2002.

[25] F. J. MacWilliams and N. J. A. Sloane, The Theory of Error-Correcting Codes, North-Holland, Amsterdam, 1977.

[26] D. C. Marinescu and G. M. Marinescu, Classical and quantum information, Academic Press, 2012.

[27] W. J. Martin, A physics-free introduction to quantum error correcting codes, Utilitas Mathematica (2004), 133-158.

[28] M. A. Nielsen and I. L. Chuang, Quantum Computing and Quantum Information, Cambridge University Press, 2000.

[29] J. Preskill, Fault-tolerant quantum computation, arXiv preprint quant-ph/9712048 (1997).

[30] J. Preskill, Lecture notes for physics 229: Quantum information and computation, California Institute of Technology (1998).

[31] B. Schumacher, Sending entanglement through noisy quantum channels, Phys. Rev. A 54 (1996), no. $4,2614-2628$.

[32] P. W. Shor, Scheme for reducing decoherence in quantum computer memory, Phys. rev. A 52 (1995), no. 4, 2493-2496.

[33] P. W. Shor, and J. A. Smolin, Quantum error-correcting codes need not completely reveal the error syndrome, arXiv preprint quant-ph/9604006 (1996).

[34] A. M. Steane, Simple quantum error-correcting codes, Phys. Rev. A 54 (1996), no. 6, 4741-4745.

[35] A. M. Steane, Error correcting codes in quantum theory, Phys.. Rev. Letters 77 (1996), no. 5, 793-797.

[36] W. G. Unruh, Maintaining coherence in quantum computers, Phys. Rev. A 51 (1995), no. 2, 992-997.

[37] J. Watrous. Theory of quantum information, lecture notes, University of Waterloo (2008). 
[38] M. M. Wilde, From classical to quantum Shannon theory, arXiv preprint arXiv:1106.1445 (2011).

[39] M. M. Wolf, Quantum channels \& operations: Guided tour, Lecture notes available at http://www-m5. ma. tum. de/foswiki/pub M 5 (2012).

[40] W. Wootters and W. Zurek, A Single Quantum Cannot be Cloned, Nature 299 (1982), 802-803.

[41] P. Zanardi and M. Rasetti, Noiseless quantum codes, Phys. Rev. Lett. 79 (1997), no. 17, 33063309. 